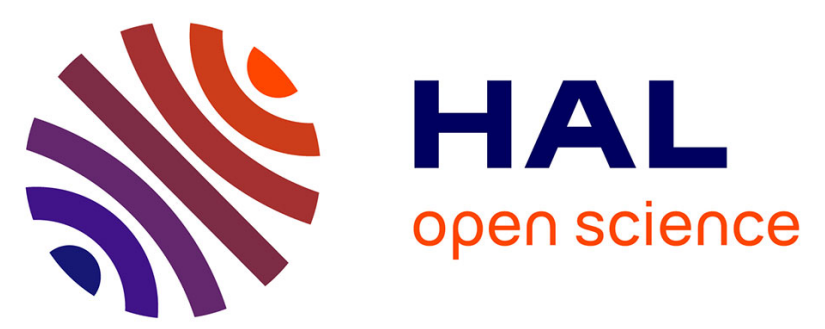

\title{
Assessment of Computational Fluid Dynamics Capabilities for the Prediction of Three-Dimensional Separated Flows: The DELFT 372 Catamaran in Static Drift Conditions
}

R. Broglia, S. Zaghi, E. Campana, T. Dogan, H. Sadat-Hosseini, F. Stern, P. Queutey, Michel Visonneau, E. Milanov

\section{To cite this version:}

R. Broglia, S. Zaghi, E. Campana, T. Dogan, H. Sadat-Hosseini, et al.. Assessment of Computational Fluid Dynamics Capabilities for the Prediction of Three-Dimensional Separated Flows: The DELFT 372 Catamaran in Static Drift Conditions. Journal of Fluids Engineering, 2019, 141 (9), 10.1115/1.4042752 . hal-02391351

\section{HAL Id: hal-02391351 \\ https://hal.science/hal-02391351}

Submitted on 26 Oct 2020

HAL is a multi-disciplinary open access archive for the deposit and dissemination of scientific research documents, whether they are published or not. The documents may come from teaching and research institutions in France or abroad, or from public or private research centers.
L'archive ouverte pluridisciplinaire HAL, est destinée au dépôt et à la diffusion de documents scientifiques de niveau recherche, publiés ou non, émanant des établissements d'enseignement et de recherche français ou étrangers, des laboratoires publics ou privés. 


\title{
Assessment of Computational Fluid Dynamics Capabilities for the Prediction of Three-Dimensional Separated Flows: The DELFT 372 Catamaran in Static Drift Conditions
}

\author{
R.Broglia1, S. Zaghi and E.F. Campana \\ CNR-INM2 - National Research Council, Institute of Marine Engineering, Rome, Italy \\ E-mail: riccardo.broglia@cnr.it; stefano.zaghi@cnr.it; emiliofortunato.campana@cnr.it \\ T.Dogan and F. Stern \\ IIHR - Iowa Institute of Hydraulic Research \\ Hydroscience and Engineering, University of Iowa, USA \\ E-mail: timur-dogan@uiowa.edu; mailto:frederick-stern@uiowa.edu \\ H.Sadat-Hosseini \\ Mechanical and Energy Engineering Department \\ University of North Texas, USA \\ E-mail: hamid.sadat@unt.edu \\ P.Queutey and M. Visonneau \\ Fluid Mechanics Laboratory \\ CNRS/ECN — Centre National de la Recherche Scientifique/Ecole Centrale Nantes, France \\ E-mail: patrick.queutey@ec-nantes.fr; Michel.Visonneau@ec-nantes.fr \\ E.Milanov \\ BSHC—Bulgarian Ship Hydrodynamics Centre, Varna, Bulgaria \\ E-mail: e.milanov@bshc.bg
}

\begin{abstract}
In this paper, capabilities of state-of-the-art computational fluid dynamics (CFD) tools in the prediction of the flow-field around a multihull catamaran advancing in straight ahead motion at nonzero drift angles are investigated. CFD estimations have been provided by three research institutes by using their in-house codes: CNR-INM using Xnavis, IIHR using CFDShip-Iowa, and CNRS/ECN using ISIS. These allowed an in-depth comparison between different methodologies, such as structured overlapping grids versus unstruc-tured grid, different turbulence models and detached eddy simulations (DES) approaches, and level-set (LS) versus volume of fluid (VoF). The activities were pursued within the NATO AVT-183 group "reliable prediction of separated flow onset and progression for air and sea vehicles," aimed at the assessment of CFD predictions of large three-dimensional separated flows. Comparison between estimations is provided for both inte-gral and local quantities, and for wave-induced vortices. Validation is reported by comparison against the available experimental fluid dynamics (EFD) data. Generally, all the simulations are able to capture the main features of the flow field; grid resolution effects are dominant in the onset phase of coherent structures and turbulence model affects the dynamic of the vortices. Hydrodynamic loads are in agreement between the submissions with standard deviation of about $3.5 \%$ for the resistance prediction and about $7 \%$ for lateral force and yaw moment estimation. Wave-induced vortices are correctly captured by both LS and VoF approaches, even if some differences have been highlighted, LS showing well-defined and long life vortices.
\end{abstract}




\section{Introduction}

The flow field around sea and air vehicles operating in offdesign conditions, such as during thigh maneuvers, is characterized by the presence of large separated flows; this, in addition to the generation of large vortical structures interacting with control surfaces, could strongly affect the stability and controllability of the vehicle. Therefore, its accurate prediction is deemed of paramount importance. To this aim, computational fluid dynamics (CFD) approach has been demonstrated to be a valuable tool for in-depth studies, even for such challenging problem. The capabilities of the state-of-the-art solvers for the prediction of steady and nonsteady (gentle) maneuvers and the advancement in (not rough) sea ways are well summarized in the latest workshops on verification and validation of ship maneuvering simulation methods [1,2] and in the latest CFD workshops on numerical ship hydrodynamics [3,4]. In Ref. [5], an in-depth review on the capabilities of hydrodynamic tools for naval design is reported. In SIMMAN 2008 [1], a good agreement (and low uncertainty) between numerical computations and measurements has been reported for static maneuvers and for captive (prescribed) maneuvers at low and medium drift angles and/or yaw rates. Comparison errors $(E)$ for the estimations of forces and moments were bounded by $5 \%$ of the experimental data $(D)$. On the contrary, rather large comparison errors $(E$ around $10 \% D)$ have been reported for maneuvers at higher drift angles and/or large yaw rates. Similar conclusions were reached in the following Workshop, the SIMMAN 2014 [2].

In the Gothenburg 2010 Workshop [3], tests were focused on straight-ahead advancement of the naval combatant 5415 and the commercial tanker KVLCC2. Overall, a very good estimation has been observed for the resistance predictions, with a negligible value for the mean comparison error (around $0.1 \% D$ ) and with very low standard deviation among numerical computations $(2.1 \% D)$. The largest mean error (under prediction of $2.0 \% D$ ) has been reported for the KVLCC2, whereas the largest scattered among the numerical estimations has been observed for the 5451 (standard deviation around 3.2\%). The agreement between computations showed a significant improvement with respect to the previous CFD Workshops. Overall conclusions highlighted that anisotropic turbulence models provide better results than standard isotropic models, even if the inspection of the flow fields and the comparison with experimental data showed an excessive dissipative behavior. However, it has been generally concluded that grid resolutions were not enough to correctly evaluate different turbulence models and, therefore, no firm conclusions could be reached. From the experimental side, data were highlighted to be too sparse and dated; moreover, scarce information was given for turbulence quantities (root-mean-square (RMS) of fluctuations, turbulent kinetic energy, etc.).

Similar conclusions were reported for the resistance estimation at the latest Tokyo 2015 Workshop [4]. In terms of turbulence models reliabilities, of particular interest was the analysis of the flow around the Japan bulk carrier. An in-depth analysis of the computational results was pursued during the workshop; the analysis indicated that, due to the relatively high block coefficient, flow field prediction in the stern of the Japan bulk carrier hull is rather challenging. Questions about the relative role played by discretization and modeling errors were raised but no conclusion was firmly established. Post Workshop analysis [6] was made for additional unsteady Reynoldsaveraged Navier-Stokes (URANS) and detached eddy simulations (DES) performed on numerical grids composed of up to $66 \mathrm{M}$ grid points. URANS results showed a rather accurate prediction of the mean axial velocity along the vortex core, but a significant underprediction for the turbulent kinetic energy (TKE); DES results provided results in better agreement with experimental fluid dynamics (EFD) for both axial velocity and TKE. However, also for this test case, due to the lack of valuable and well detailed measurements, validation assessment for the simulations was not possible.

The aim of the NATO AVT 183 working group on "reliable prediction of separated flow onset and progression for air and sea vehicles" is the assessment of the prediction capabilities of stateof-the-art ship hydrodynamics solvers in the presence of large separated flows and three-dimensional vortex separations. For the sea facet, the collaborative effort involved nine institutions among ten different countries, with a total of 33 CFD contributions [7]. Experimental and computational activities have been focused on three benchmark cases:

- The surface combatant 5415 advancing straight ahead and with $0 \mathrm{deg}$ and $20 \mathrm{deg}$ of drift angle for which experimental data and a summary of the CFD predictions can be found in Refs. [8] and [9], respectively.

- The KVLCC2 tanker, with experimental tests conducted in wind tunnel and in shallow water for 30 deg drift angle [10].

- A high-speed multihull Delft Catamaran 372 (DC) advancing in steady drift for which an overview of the CFD capabilities is provided here (detailed results can be found in Ref. [11]).

For the DC 372 catamaran model, several experimental and numerical research works are available in the literature. Maneuverability and stability properties in both deep and shallow water have been studied (by means of combined EFD and CFD approaches) in Refs. [12-15]. Resistance and self-propulsion tests (water-jets) have been reported in Refs. [16] and [17], whereas sea keeping studies were provided in Refs. [18] and [19]. Interference effects between the two hulls of the catamaran were deeply investigated in Refs. [20-23]. Free running tests in deep and shallow water have been reported in Ref. [24].

The present work focuses on the analysis of the numerical methods, grid strategy/resolution, turbulence models, and air/ water interface modeling. The assessment of CFD capabilities is provided for the onset and progression of vortex structures, axial velocity and vorticity, TKE, wave-induced vortices, wave patterns, and hydrodynamic loads. The operative conditions include two speeds of advancement $(\mathrm{Fr}=0.4$ and 0.5$)$ and two drift angles ( $6 \mathrm{deg}$ and $9 \mathrm{deg}$ ). The available experimental data include trim and sinkage measurements and velocity maps (by using a StereoPIV system) on several transversal planes from CNR-INM [25] and hydrodynamic loads by BSHC $[15,24]$. Additional simulations at different conditions have been also provided; in the following, also these simulations will be discussed and validated against the available experimental data.

\section{Test Conditions and Submissions}

2.1 Catamaran Model and Test Conditions. The highspeed Delft Catamaran 372 (DC) [26,27] has been selected as benchmark vessel. The model is featured by a couple of symmetrical hulls; main particulars and body plane are reported in Table 1 and Fig. 1. Each demi-hull is characterized by a sharp bow edge and a transom stern. Several geo-sym models have been built and tested. CNR-INM built a fiberglass model (CNR-INSEAN model $2554)$ at the same scale as the Delft one $\left(L_{p p}=3.0 \mathrm{~m}\right)$; this model has been used for sinkage and trim measurements at several drift angle and for S-PIV velocity measurements. BSHC built two models: the M0390 (with length $L_{p p}=3.632 \mathrm{~m}$ ) and the M0412 $\left(L_{p p}=3.993 \mathrm{~m}\right)$. Main particulars of these three models are reported in Table 1. CNR-INM model was equipped with turbulence stimulator device consisting of cylindrical studs placed along a line, $30 \mathrm{~mm}$ spaced; the cylinders have dimensions of $4 \mathrm{~mm}$ in height and $3 \mathrm{~mm}$ in diameter. The stimulator device is placed $70 \mathrm{~mm}$ downstream of the bow. No turbulence stimulator device has been used by BSHC.

2.2 Computational Fluid Dynamics Submissions. Numerical results have been submitted by three different organizations from three countries; each research group has used its own inhouse CFD tools, several turbulence models, and different grids (see Table 2). The submissions are summarized in Table 3. Numerical simulations have been pursued with sinkage and trim 
Table 1 Main particulars of the catamaran models

\begin{tabular}{|c|c|c|c|c|}
\hline Dimension & Symbol & INSEAN 2554 & BSHC M0390 & BSHC M0412 \\
\hline Length between perpendiculars & $L_{\mathrm{pp}}$ & $3.000 \mathrm{~m}$ & $3.627 \mathrm{~m}$ & $3.993 \mathrm{~m}$ \\
\hline Beam overall & $B / L_{\mathrm{pp}}$ & & 0.313 & \\
\hline Beam demi hull & $b / L_{\mathrm{pp}}$ & & 0.080 & \\
\hline Distance between center of hulls & $H / L_{\mathrm{pp}}$ & & 0.233 & \\
\hline Draft & $T / L_{\mathrm{pp}}$ & & 0.050 & \\
\hline Displacement & $\Delta / L_{\mathrm{pp}}^{\mathrm{Pr}_{3}}$ & & $3.225 \times 10^{-3}$ & \\
\hline Vertical CoG & $\mathrm{KG} / L_{\mathrm{pp}}$ & 0.113 & - & - \\
\hline Longitudinal CoG & LCG & & 0.470 & \\
\hline
\end{tabular}
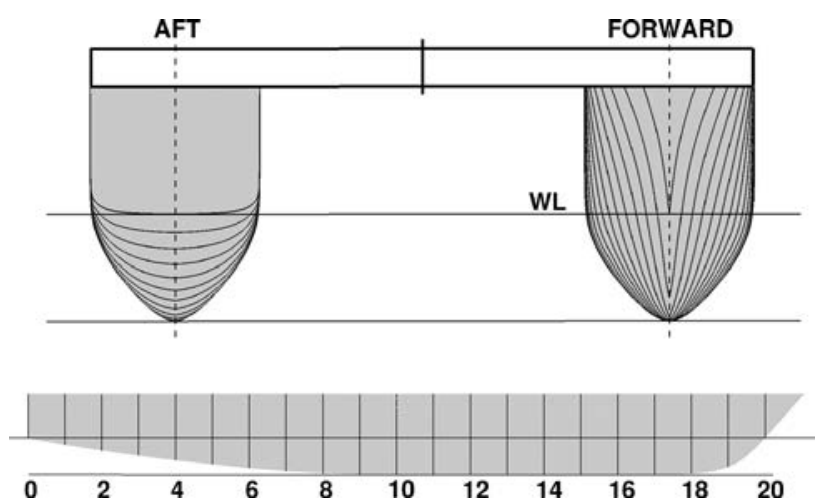

Fig. 1 Basic geometry definition and body plan of the Delft 372 catamaran model

fixed at the dynamical positions; values were taken from ad hoc experiments conducted at CNR-INM [25]. Froude and Reynolds numbers correspond to a model with length between perpendiculars equal to $3 \mathrm{~m}$, i.e., to a model size equal to the one tested at CNR-INM (main particulars are reported in Table 1). These conditions are considered mandatory for the CFD assessment; some additional computations, at different operative conditions, have been provided by IIHR. These simulations will be also described and discussed in the following.

Hereafter, computations will be denoted as follows:

(1) IIHR-tur-grid, computations from IIHR (using CFDShipIowa), with tur denoting the turbulence model (explicit algebraic Reynolds stress model (EARSM), shear stress transport (SST), or DES) and grid the grid used (G2, G3, G4, G5, GW, GR, or GO);

(2) Xnavis-SA, CNR-INM simulations (Xnavis code); and

(3) ISIS-tur results from CNRS/ECCN group, using ISIS code, tur denoting the turbulence model employed (EARSM, $k \omega$ Spalart-Allmaras, Inviscid); with ISIS-tur-automatic mesh refinement (AMR) indicating those computations performed using AMR capabilities.

Main characteristics of the numerical solvers, including information about the turbulence model employed and the numerical grid adopted by different institutions, are summarized in Secs. 2.2.1-2.2.3
2.2.1 Xnavis: CNR-INM. CNR-INM provided numerical simulations by using the Xnavis CFD code; the solver is a general purpose unsteady Navier-Stokes equations solver. The main characteristics of the solver are reported here; for more details and some applications, the reader is referred to Refs. [28-33].

Numerical discretization of the URANS equations is achieved in the framework of a finite volume formulation with conservative variables at cell centers. The computation of the convective fluxes and the surface integral of the velocity in the continuity equation can be done by several approximation schemes available in the code, ranging from the first-order Godunov scheme, the secondorder total variation diminishing scheme, the third-order essentially nonoscillatory scheme, the third-order weighted essentially non oscillatory scheme, and the classical fourth-order centered scheme (for more details, see Refs. [28] and [30]). Viscous fluxes are discretized by means of the classical finite volumes secondorder formulation. Momentum and mass conservation equations are coupled by a pseudo-time integration that exploits an Euler implicit scheme with approximate factorization; local pseudo time-step and an efficient multigrid method [34] are used to accelerate the convergence toward the divergence-free solution. In the solver, several turbulence models have been implemented, however, computations performed around the catamaran have been pursued using the one-equation Spalart-Allmaras [35] model. This model has been chosen because it has been demonstrated to be a good compromise between accuracy and computational overhead (which keeps small being the model requiring only a one additional transport equation). However, since the model has been also demonstrated to be rather dissipative, special attention must be posed in generating the computational mesh, especially where high velocity gradients are expected. The free surface effects are simulated through a fully nonlinear level-set (LS) single phase methodology $[29,33]$. The numerical mesh employed is based on "chimera" grid technique. In this approach, the possibility to let the grids overlap is achieved through a modification of both the boundary conditions and internal point treatment for those zones where overlapping appears: dynamic overlapping grids method allows for easy and accurate handling of complex geometries and multiple bodies in relative motion. High performance computing capabilities are achieved by an efficient shared and distributed memory parallelization [32].

For the simulations presented here, computational domain extends about $1.5 L_{p p}$ in front, $3.5 L_{p p}$ rear, $2.0 L_{p p}$ on the side, and

Table 2 Summary of Institutes and codes

\begin{tabular}{lccc}
\hline Institute & Code & Turbulence model & Grid \\
\hline CNR-INM & Xnavis & Spalart-Allmaras & $10 \mathrm{M}\left(y^{+} \leq 1\right)$ \\
IIHR & CFDship-IOWA & EARSM & G2 $\left(124 \mathrm{M}, y^{+}=0.21\right), \mathrm{G} 3\left(44 \mathrm{M}, y^{+}=0.30\right), \mathrm{G} 4$ \\
& & $\mathrm{SST}$ & $\left(15.5 \mathrm{M}, y^{+}=0.45\right), \mathrm{G} 5\left(5.5 \mathrm{M}, y^{+}=0.66\right) \mathrm{GW}\left(51 \mathrm{M}, y^{+}=0.30\right)$, \\
ECN/CNRS & DES & $\mathrm{GR}\left(13 \mathrm{M}, y^{+}=0.30\right), \mathrm{GO}\left(81 \mathrm{M}, y^{+}=0.21\right)$ \\
& ISIS & EARSM & $20 \mathrm{M}\left(y^{+}=30\right), 24 \mathrm{M}\left(y^{+} \leq 1\right), \mathrm{MMR}\left(y^{+}=30\right)$ \\
& & $k \omega$ & \\
& & Spalart-Allmaras Inviscid (Euler) & \\
& &
\end{tabular}


Table 3 Summary of submissions

\begin{tabular}{|c|c|c|c|c|c|c|}
\hline $\mathrm{Fr}$ & $\operatorname{Re}$ & $\beta$ & DOF & CNR-INM & IIHR & CNRS/ECN \\
\hline 0.40 & $5.72 \times 10^{6}$ & $6 \mathrm{deg}$ & $\mathrm{FX}_{\sigma \tau}$ & $\mathrm{SA}-10 \mathrm{M}\left(y^{+} \approx l\right)$ & $\begin{array}{c}\text { SST-G3 }(44 \mathrm{M}) \\
\text { EARSM-G3 }(44 \mathrm{M}) \\
\text { SST-GO }(81 \mathrm{M}) \\
\text { EARSM-GO }(81 \mathrm{M})\end{array}$ & $\begin{array}{c}\text { EARSM, AMR }(\sim 2.9 \mathrm{M}) \\
\text { EARSM, 20M } \\
\kappa \omega, \sim 20 \mathrm{M} \\
\kappa \omega, \operatorname{AMR}(\sim 2.9 \mathrm{M})\end{array}$ \\
\hline 0.40 & $5.72 \times 10^{6}$ & $9 \mathrm{deg}$ & $\mathrm{FX}_{\sigma \tau}$ & $\mathrm{SA}-10 \mathrm{M}\left(y^{+} \approx 1\right)$ & SST-G3 (44M) & $\operatorname{EARSM}, \operatorname{AMR}(\sim 4,7 \mathrm{M})$ \\
\hline 0.50 & $7.15 \times 10^{6}$ & $6 \mathrm{deg}$ & $\mathrm{FX}_{\sigma \tau}$ & $\mathrm{SA}-10 \mathrm{M}\left(y^{+} \approx l\right)$ & $\begin{array}{l}\text { SST-G3 (44M) } \\
\text { SST-GR (13M) }\end{array}$ & EARSM, $\operatorname{AMR}(\sim 4.1 \mathrm{M})$ \\
\hline 0.50 & $7.15 \times 10^{6}$ & $9 \mathrm{deg}$ & $\mathrm{FX}_{\sigma \tau}$ & $\mathrm{SA}-10 \mathrm{M}\left(y^{+} \approx 1\right)$ & $\begin{array}{l}\text { SST-G3 (44M) } \\
\text { SST-GW (51M) }\end{array}$ & $\begin{array}{c}\text { EARSM, AMR }(\sim 6.4 \mathrm{M}) \\
\text { EARSM, 20M } \\
\kappa \omega, \text { AMR }(\sim 6.6 \mathrm{M}) \\
\kappa \omega, \sim 20 \mathrm{M} \\
\kappa \omega, \sim 24 \mathrm{M} \\
\text { SA, } \sim 24 \mathrm{M} \\
\text { Euler, } \sim 20 \mathrm{M}\end{array}$ \\
\hline 0.50 & $7.15 \times 10^{6}$ & $6 \mathrm{deg}$ & $\mathrm{FX}_{0}$ & SA-10M $\left(y^{+} \approx 1\right)$ & $\begin{array}{c}\text { SST-GR }(13 \mathrm{M}) \\
\text { SST-DES-G5 }(5.5 \mathrm{M}) \\
\text { SST-DES-G4 }(15.5 \mathrm{M}) \\
\text { SST-DES-G3 }(44 \mathrm{M}) \\
\text { SST-DES-G2 }(124 \mathrm{M})\end{array}$ & None \\
\hline
\end{tabular}

Note: In the table, $\mathrm{FX}_{\sigma \tau}$ and $\mathrm{FX}_{0}$ stand for fixed sinkage and trim at the dynamical conditions and fixed at even keel.

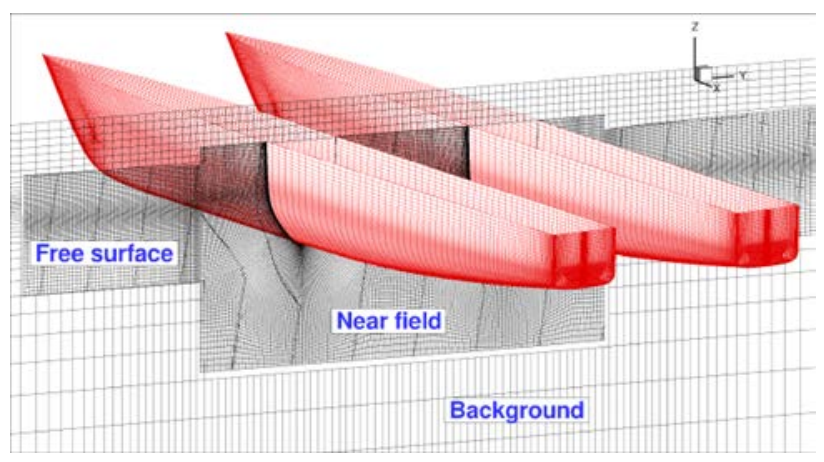

Fig. 2 Overview of the overlapping grid used by Xnavis-SA computations
$1.0 L_{p p}$ in depth. The computational mesh consists of about $10.6 \mathrm{M}$ of control volumes distributed among 200 adjacent and overlapping blocks. Structured blocks are divided into three groups (see Fig. 2): one is composed of blocks body-fitted to the hulls and it is used to describe both the boundary layer region and the near field; most of the vertical structures shed from the hulls are captured by this group of blocks. The desired drift angle and attitude is obtained rotating this group of blocks which is rigidly fixed with the hulls of the catamaran. The resolution is such that to guarantee a height of the first cell equal to one in wall units; the main vortex is discretized with about 20 points. The second group of blocks is used to refine the region around the free surface; about $1.8 \mathrm{M}$ control volumes are distributed among these blocks. The last group of blocks is composed of the background grid. As what boundary conditions concern, no-slip boundary conditions are enforced on the hull surface; extrapolation conditions are imposed on the top,

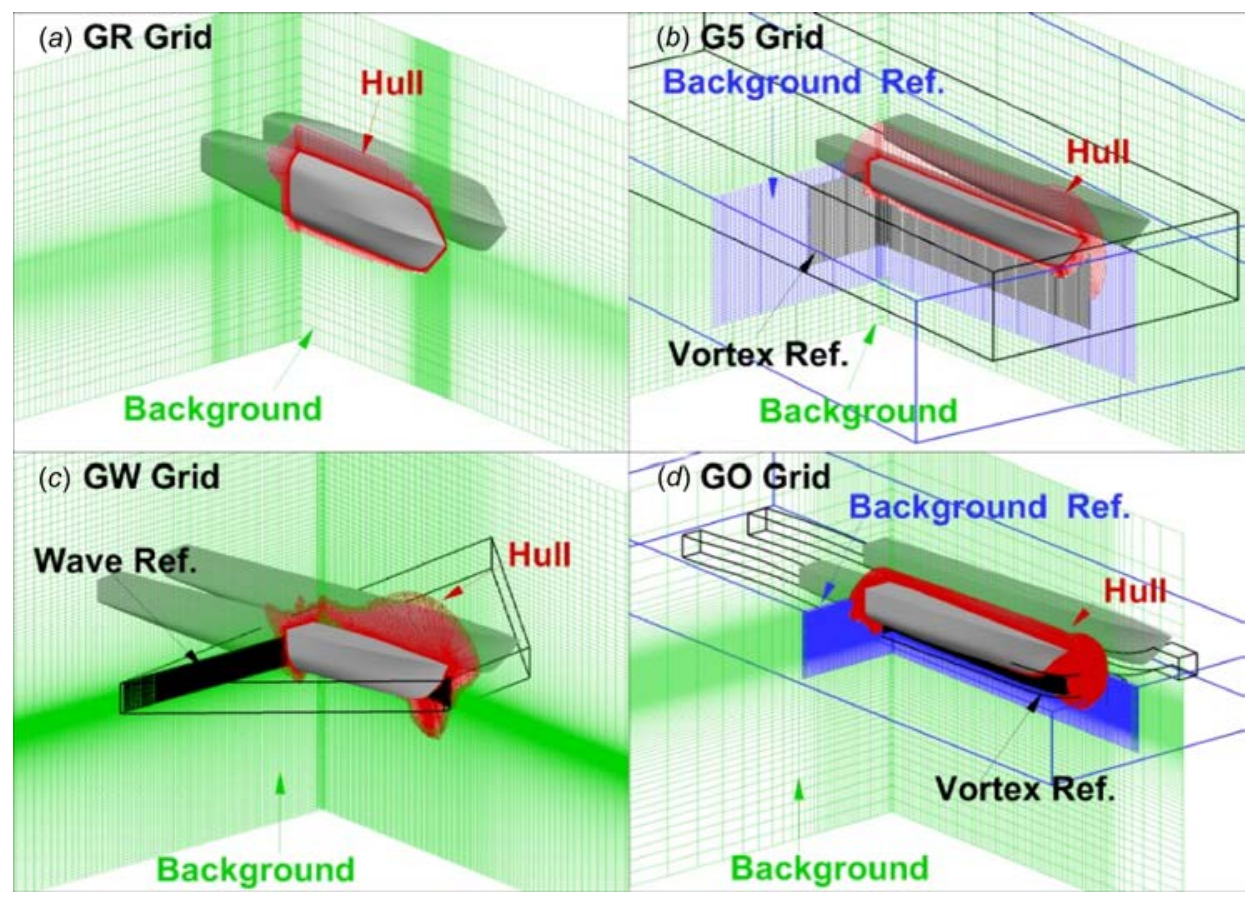

Fig. 3 Comparison of grid designs used by CFDShip-lowa (GR, G5, GW, and GO) 
Table 4 Grid design for IIHR CFDShip-lowa

\begin{tabular}{|c|c|c|c|c|c|c|c|c|c|}
\hline \multirow[b]{2}{*}{ Variable } & & \multicolumn{8}{|c|}{ Grid name } \\
\hline & & G5 & G4 & G3 & $\mathrm{G} 2$ & G1 & GO & GR & GW \\
\hline \multirow[t]{5}{*}{ Block name } & Hull & 829,926 & $2,347,385$ & $6,639,408$ & $18,779,082$ & $53,115,264$ & $44,262,720$ & $6,639,408$ & $6,639,408$ \\
\hline & Background & $1,500,000$ & $4,229,718$ & $12,000,000$ & $33,957,736$ & $96,000,000$ & $4,191,750$ & $6,572,104$ & $23,329,152$ \\
\hline & Background Ref. & $1,250,000$ & $3,543,894$ & $10,000,000$ & $28,211,421$ & $80,000,000$ & $19,468,750$ & - & - \\
\hline & Vortex/Wave Ref. & $1,890,000$ & $5,297,880$ & $15,120,000$ & $42,723,153$ & $120,960,000$ & $13,032,864$ & - & $21,000,000$ \\
\hline & Total & $5,469,926$ & $15,418,877$ & $43,759,408$ & $123,671,392$ & $350,075,264$ & $80,956,084$ & $13,211,512$ & $50,968,560$ \\
\hline \# Blocks & & 4 & 4 & 4 & 4 & 4 & 4 & 2 & 3 \\
\hline$y^{+}$ & & 0.66 & 0.45 & 0.3 & 0.21 & 0.15 & 0.3 & 0.3 & 0.3 \\
\hline
\end{tabular}

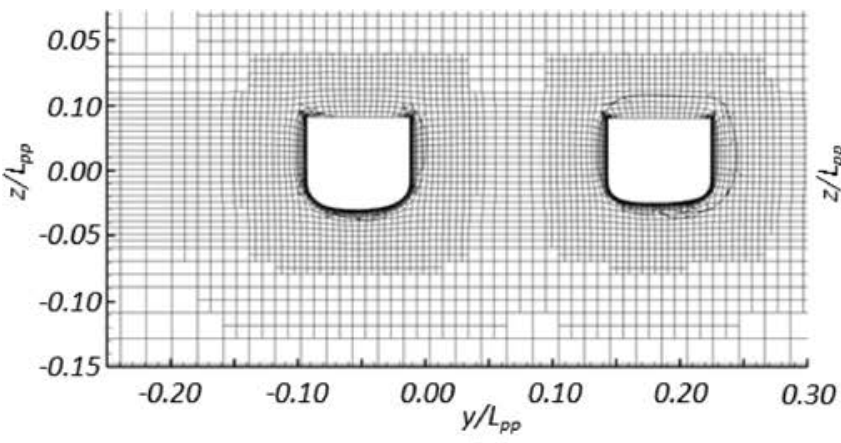

(a)

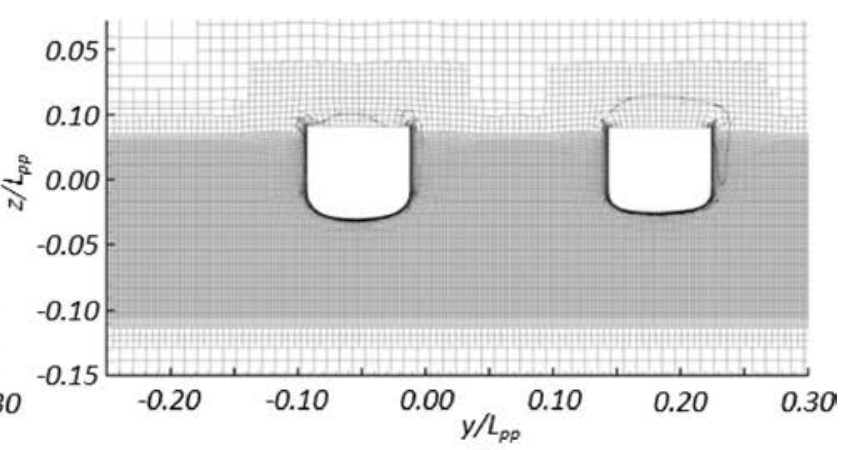

(b)

Fig. 4 Cross-section of the ISIS grid at the transversal plane $\left(x / L_{p p}=0.95\right.$ from the forward perpendicular): (a) original grid for AMR and (b) fine grid

Table 5 PMM steady drift test matrix

\begin{tabular}{lccc}
\hline Fr & Re & $\beta(\mathrm{deg})$ & Repeatability \\
\hline $0.20-0.70 \Delta=0.1$ & $2.86 \times 10^{5}-1.00 \times 10^{7}$ & 0 deg, 3 deg, 6 deg, 9 deg & Fr $=0.2,0.3,0.4,0.5 \beta=6$ deg, 9 deg
\end{tabular}

bottom, and sides of the domain; inlet boundary conditions are imposed on the front; and finally, zero gradient conditions are imposed at the outflow.

2.2.2 Computational Fluid Dynamics Ship-Iowa: IIHR, The University of Iowa. Numerical simulations provided by IIHR, The University of Iowa, have been made by the in-house CFDShipIowa V4.5, which is an incompressible URANS/DES solver [36] for ship hydrodynamics applications. Several turbulence models have been implemented in the solver, including the isotropic blended $k \varepsilon / k \omega$, the anisotropic EARSM, the $k \omega$-SST model, and DES approaches with near-wall models. For the air/water interface, CFDShip-Iowa V4.5 uses a single-phase level set approach. The code has the capability of running full six degrees-of-freedom (DOFs) simulations. The numerical methods are based on a finite difference discretization on body-fitted curvilinear grids; viscous terms are discretized by a second-order centered schemes, whereas higher-order upwind schemes have been used for the convection terms. Time integration is achieved by a second-order backward difference Euler scheme. High-performance parallel computing capabilities are obtained by a message passing interface-based domain decomposition approach; each decomposed block is mapped to one processor. The overset domain connectivity information which is used to connect the solution between overlapping grids is obtained using the code SUGGAR [37].

For the computations around the catamaran, a rectangular computational domain has been used, with extensions $\left[0.4 L_{p p}\right.$, $\left.2.6 L_{p p}\right],\left[-0.7 L_{p p}, 0.7 L_{p p}\right]$, and $\left[1.3 L_{p p}, 1.3 L_{p p}\right]$ in the streamwise, spanwise, and vertical directions, respectively. No-slip boundary conditions are enforced on the catamaran surfaces, whereas far-field boundary conditions are imposed on the top, the bottom, and the sides of the domain; at the inflow section, inlet boundary condition is used with zero pressure gradient. Extensive $\mathrm{V} \& \mathrm{~V}$ study was conducted on Delft catamaran for a wide range of Froude numbers $(0.3 \leq \mathrm{Fr} \leq 0.77)$ and drift angles $(0 \mathrm{deg} \leq \beta \leq$ $30 \mathrm{deg}$ ); results are reported in Refs. [38] and [39]. Based on the previous study, two different grids with different overset topology and refinement blocks were designed, namely the GW (which

Table 6 Sinkage and trim at $\mathrm{Fr}=0.4,0.5$ and $\beta=6 \mathrm{deg}, 9 \mathrm{deg}$

\begin{tabular}{|c|c|c|c|c|c|c|}
\hline \multirow[b]{2}{*}{$\beta(\operatorname{deg})$} & \multirow[b]{2}{*}{ Fr } & \multirow[b]{2}{*}{$\operatorname{Re}$} & \multirow[b]{2}{*}{$\sigma / L_{\mathrm{pp}}$} & \multirow[b]{2}{*}{$\tau(\operatorname{deg})$} & \multicolumn{2}{|c|}{$\mathrm{U}_{\mathrm{D}}[\% \mathrm{D}]$} \\
\hline & & & & & $U_{\sigma}(\% \sigma)$ & $U_{\tau}(\% \tau)$ \\
\hline $6 \mathrm{deg}$ & $\begin{array}{l}0.40 \\
0.50\end{array}$ & $\begin{array}{l}5.72 \times 10^{6} \\
7.15 \times 10^{6}\end{array}$ & $\begin{array}{l}-5.213 \times 10^{-3} \\
-6.886 \times 10^{-3}\end{array}$ & $\begin{array}{l}0.35 \mathrm{deg} \\
1.71 \mathrm{deg}\end{array}$ & $\begin{array}{l}2.53 \% \\
2.96 \%\end{array}$ & $\begin{array}{l}3.63 \% \\
1.15 \%\end{array}$ \\
\hline $9 \mathrm{deg}$ & $\begin{array}{l}0.40 \\
0.50\end{array}$ & $\begin{array}{l}5.72 \times 10^{6} \\
7.15 \times 10^{6}\end{array}$ & $\begin{array}{l}-6.091 \times 10^{-3} \\
-8.653 \times 10^{-3}\end{array}$ & $\begin{array}{l}0.19 \mathrm{deg} \\
1.51 \mathrm{deg}\end{array}$ & $\begin{array}{l}2.93 \% \\
2.51 \%\end{array}$ & $\begin{array}{l}6.00 \% \\
1.57 \%\end{array}$ \\
\hline
\end{tabular}

Note: EFD data from CNR-INM. 
consists of a total of $51 \mathrm{M}$ grid points) and the G1 (which counts $350 \mathrm{M}$ grid points). The size of the refinement block for $\mathrm{G} 1$ was determined based on the vortical structures of the tests at the larger drift angle tested $(\beta=30 \mathrm{deg})$ to guarantee that all the vortical structures were captured for the whole range of drift angles simulated. Grid G1 was coarsened with a refinement ratio of $\sqrt{2}$ to create grids, G2-G5. An overview of the grids used is given in Fig. 3. The block-structured overlapped grids used by IIHR can be summarized as follows (see Table 4): grid GR (13M), which is the base grid without any refinement blocks. G5 (5.5M), G4 (15.5M), G3 (44M), and G2 (124M), which are successive grid refining with refinement ratio of $\sqrt{2}$; the triplets G5-G4-G3 and G4-G3-G2 have been used for verification purposes on grids $\mathrm{G} 3$ and $\mathrm{G} 2$, respectively. Grid GW (50M) is similar to G3 (44M) with a refinement block around the free surface. Finally, grid GO $(81 \mathrm{M})$ is similar to $\mathrm{G} 1(350 \mathrm{M})$ with a refinement block along the vortex core. In the simulations presented here, no simulation has been done on the finest grid, i.e., on G1.

2.2.3 ISIS: $C N R S / E C N$. CNRS/SCN computations have been pursued using ISIS solver, which is an incompressible URANS tool mainly designed for applications in marine hydrodynamics framework. The tool is available as a part of the FINETM/ Marine computing suite. The method features several turbulence models with different levels of sophistication: the classical twoequation $k \varepsilon$ and $k \omega$ models, the anisotropic two-equation EARSM, and the Reynolds stress transport models $k \omega$ Menter's model [40] $(k \omega$-SST) with or without rotation corrections are available [41,42]. Wall function or low-Reynolds near wall formulations can be used with models. Hybrid large eddy simulation (LES) turbulence models based on DES are also implemented in the tool; this approach has been already validated in automotive flows characterized by large separations [43]. For what propeller simulations concerns, it has to be highlighted that several cavitation models are available.

The solver is based on an unstructured (face-based) finite volume discretization of the governing equations. Unknown state variables are cell-centered, whereas the systems of equations used in the implicit time stepping procedure are constructed face by face; fluxes are computed on each face and it is added to the two cells that share the face. This technique poses no specific requirements on the topology of the cells, allowing the use of completely unstructured grids; moreover, cells with an arbitrary number of arbitrarily shaped faces can be used. Pressure-velocity coupling is enforced through a Rhie and Chow SIMPLE type method. Air/ water interface is modeled with a multiphase flow approach: the water surface is captured with a conservation equation for the volume fraction of water, discretized with specific compressive discretization schemes [44]. Six degrees-of-freedom capabilities are described in Ref. [45]. Time integration of Newton's law for the rigid body motion is combined with analytical weighted or elastic analogy grid deformation to adapt the fluid mesh to the moving ship. Relative motions of appendages, propellers, or bodies are handled by a suitable sliding grid approach. Propellers can be modeled using actuator disc theory, by a URANS/boundary element method coupling approach [46], or with direct discretization through, e.g., the rotating frame method or sliding interface approaches [47]. Finally, in the code, an efficient anisotropic AMR procedure has been implemented; several flow-related criteria can be used to guide mesh refinement [48].

The size of the computational domain is based on the dimensions of the CNR-INM towing tank, i.e., dimensions are $6.60 \times 13.50$ in meters for the depth and the width, respectively, and it extends $-26.5 \mathrm{~m}$ forward and $15 \mathrm{~m}$ downward with the origin at the aft perpendicular. The required drift angle is achieved rotating the catamaran; in this way, the external boundary conditions do not depend on the drift angle. The enforced boundary conditions are far field Dirichlet condition on the inflow, prescribed pressure on the outflow, and no-slip condition on the side faces (which approximate the walls of the towing tank). Pressure

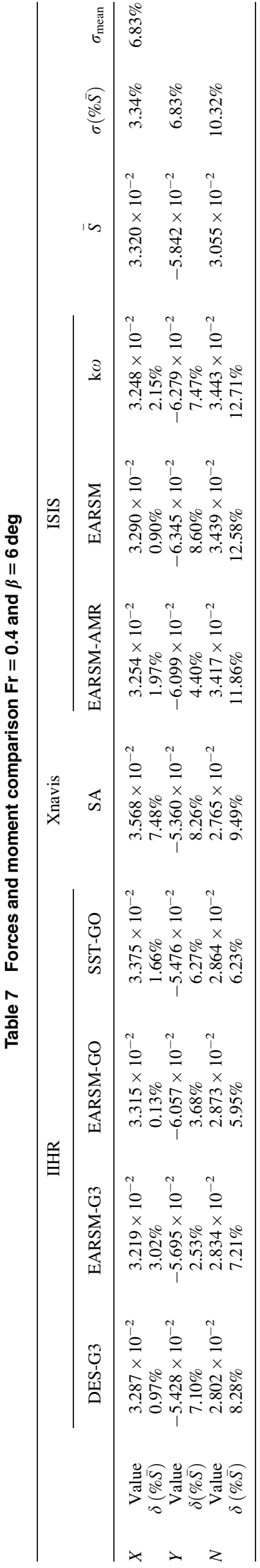


Table 8 Forces and moment comparison $\mathrm{Fr}=0.4$ and $\beta=9$ deg

\begin{tabular}{|c|c|c|c|c|c|c|c|}
\hline & & $\begin{array}{c}\text { IIHR } \\
\text { DES-G3 }\end{array}$ & $\begin{array}{l}\text { Xnavis } \\
\text { SA }\end{array}$ & $\begin{array}{c}\text { ISIS } \\
\text { EARSM-AMR }\end{array}$ & $\bar{S}$ & $\sigma(\% \bar{S})$ & $\sigma_{\text {mean }}$ \\
\hline $\mathrm{X}$ & $\begin{array}{l}\text { Value } \\
\delta(\% \bar{S})\end{array}$ & $\begin{array}{l}4.564 \times 10^{-2} \\
1.64 \%\end{array}$ & $\begin{array}{l}4.836 \times 10^{-2} \\
4.22 \%\end{array}$ & $\begin{array}{l}4.521 \times 10^{-2} \\
2.58 \%\end{array}$ & $4.640 \times 10^{-2}$ & $3.68 \%$ & $3.72 \%$ \\
\hline Y & $\begin{array}{l}\text { Value } \\
\delta(\% \bar{S})\end{array}$ & $\begin{array}{l}-1.090 \times 10^{-1} \\
0.34 \%\end{array}$ & $\begin{array}{l}-1.073 \times 10^{-1} \\
1.24 \%\end{array}$ & $\begin{array}{l}-1.097 \times 10^{-1} \\
0.90 \%\end{array}$ & $-1.087 \times 10^{-1}$ & $1.11 \%$ & \\
\hline $\mathrm{N}$ & $\begin{array}{l}\text { Value } \\
\delta(\% \bar{S})\end{array}$ & $\begin{array}{l}4.948 \times 10^{-2} \\
2.64 \%\end{array}$ & $\begin{array}{l}4.848 \times 10^{-2} \\
4.60 \%\end{array}$ & $\begin{array}{l}5.450 \times 10^{-2} \\
7.25 \%\end{array}$ & $5.082 \times 10^{-2}$ & $6.35 \%$ & \\
\hline
\end{tabular}

is prescribed on top and bottom boundaries. All the grids are generated with HEXPRESS.

For the computations around the catamaran, the basic grid generated for simulations using AMR capabilities contains about $1 \mathrm{M}$ of grid cells (see Fig. 4). For the fine mesh, grid density is explicitly increased in a box surrounding the catamaran $[-1.0$, $3.4] \times[-1] \times[-0.3,0.0075]$ in meters in the $x, y$, and $z$ directions, respectively, with the origin placed at the forward perpendicular, $x$-axis positive downstream $z$-axis vertical, positive upward. When AMR is activated, starting from the original grid, the refinement on the vertical axis is acting everywhere, whereas the same box is used to restrict the longitudinal and transversal refinements. Inside the box and outside the viscous layer, the resulting grid resolution on a fine grid is $\Delta x=\Delta y=0.0025 L_{\mathrm{PP}}$ and $\Delta z=0.00125 L_{\mathrm{PP}}$. With AMR, the finest size cells are created in the vortices and close to the air-water interface; obviously, the resulting grid depends on both the numerical settings and the adopted turbulence model. For the computations reported here, negligible differences have been observed, with $\Delta x=\Delta y=0.0006-0.0012 L_{\mathrm{PP}}$ and $\Delta z=0.00120 L_{\mathrm{PP}}$. In all cases, the time-step used to reach a steady-state solution is $0.005 \mathrm{~s}$. Depending on the case, numerical simulations are carried out for $10-20 \mathrm{~s}$ in physical time, large enough to reach a steady axial force within $0.1 \%$ of the mean value. The use of AMR capabilities reduces the CPU cost by a factor 5 compared to a fine grid resolution.

\section{Force and Moments}

3.1 Available Experimental Data. Forces $(X, Y)$ and yaw moment $(N)$ were measured at BSHC at conditions different at which mandatory computations have been performed. In particular, experimental tests at BSHC have been conducted with two models with lengths between perpendiculars equal to $3.632 \mathrm{~m}$ and $3.993 \mathrm{~m}[15,24]$, whereas CFD computations were performed considering a $3 \mathrm{~m}$ model (same model, i.e., same Reynolds number considered for the S-PIV tests [25]). Moreover, the models tested at BSHC were not provided with any turbulence stimulator device. The differences in the model length and the absence of any turbulence stimulator in the experiments do not allow a rigorous comparison between CFD and EFD data. In Ref. [11], an attempt for the validation of the CFD estimations has been done; as reference data, values interpolated from hydrodynamic loads acquired at four speeds $(\mathrm{Fr}=0.15,0.25,0.45$, and 0.65$)$ and
8-10 deg drift angles for a $\mathrm{Lpp}=3.627 \mathrm{~m}$ model length were taken. Interpolation in the $\mathrm{Fr} / \beta$ plane at the four conditions at which computations have been done has been performed by using a polyharmonic spline of second order. None of the Reynolds number corrections were considered.

Unfortunately, when CFD estimations were compared against measurements, large comparison errors were observed, without any possibility to distinguish if EFD/CFD disagreements were ascribable to the difference in the conditions or to computational inaccuracies. Therefore, comparison with EFD data will not reported here; for a qualitative validation of the CFD estimations, the reader is referred to Ref. [11]. Here, the accuracy of the CFD submissions will be measured globally by the standard deviation of the estimations and individually, i.e., for each submission, by the deviation from the mean.

Experiments were also performed at CNR-INM, in the towing tank number 2, whose dimensions are $\mathrm{L} \times \mathrm{W} \times \mathrm{H}=210$ $\mathrm{m} \times 9 \mathrm{~m} \times 3.6 \mathrm{~m}$. The model employed was $3 \mathrm{~m}$ length; measurements were taken for sinkage and trim for several speeds and drift angles (test conditions are reported in Table 5). Repeatability (minimum six runs) has been provided for four Fr and two drift angles. Due to technical difficulties, hydrodynamic loads were not measured during these tests. Results (only for the conditions at which S-PIV measurements are taken) are reported in Table 6. For more details about these measurements and the whole set of results, the reader is referred to Refs. [25] and [49].

3.2 Method of Analysis. Hydrodynamic loads are referred to a system of reference with the $x$-axis aligned with the longitudinal axis of the catamaran (positive downwind), the $y$-axis lying on the undisturbed free surface pointing starboard (windward) side, and the $z$-axis vertical positive upward. The origin is placed at the forward perpendicular. The yaw moment $N$ is computed at $L_{p p} / 2$. Forces and moment are made nondimensional as

$$
X=\frac{\bar{X}}{\frac{1}{2} \rho U_{\infty}^{2} L_{p p} T} \quad Y=\frac{\bar{Y}}{\frac{1}{2} \rho U_{\infty}^{2} L_{p p} T} \quad N=\frac{\bar{N}}{\frac{1}{2} \rho U_{\infty}^{2} L_{P P}^{2} T}
$$

where $\bar{X}$ and $\bar{Y}$ are, respectively, the longitudinal (along $x$-axis) and the lateral force (along $y$-axis) in Newton, and $\bar{N}$ is the yaw moment in Newton-meter. In the previous relations, $\rho$ is the density of the water, $U_{\infty}$ is the speed of advancement, $L_{p p}$ the length

Table 9 Forces and moment comparison. $\mathrm{Fr}=0.5$ and $\beta=6 \mathrm{deg}$

\begin{tabular}{|c|c|c|c|c|c|c|c|c|}
\hline & & \multicolumn{2}{|c|}{ IIHR } & \multirow{2}{*}{$\begin{array}{c}\text { Xnavis } \\
\text { SA }\end{array}$} & \multirow{2}{*}{$\begin{array}{c}\text { ISIS } \\
\text { EARSM-AMR }\end{array}$} & \multirow[b]{2}{*}{$\bar{S}$} & \multirow[b]{2}{*}{$\sigma(\% \bar{S})$} & \multirow[b]{2}{*}{$\sigma_{\text {mean }}$} \\
\hline & & DES-G3 & EARSM-GR & & & & & \\
\hline $\mathbf{X}$ & Value $\delta(\% \bar{S})$ & $\begin{array}{l}3.874 \times 10^{-2} \\
1.08 \%\end{array}$ & $\begin{array}{l}3.516 \times 10^{-2} \\
10.24 \%\end{array}$ & $\begin{array}{l}4.284 \times 10^{-2} \\
9.38 \%\end{array}$ & $\begin{array}{l}3.993 \times 10^{-2} \\
1.94 \%\end{array}$ & $3.917 \times 10^{-2}$ & $8.12 \%$ & $7.12 \%$ \\
\hline $\mathbf{Y}$ & Value $\delta(\% \bar{S})$ & $\begin{array}{l}-5.172 \times 10^{-2} \\
2.77 \%\end{array}$ & $\begin{array}{l}-4.768 \times 10^{-2} \\
5.26 \%\end{array}$ & $\begin{array}{l}-4.964 \times 10^{-2} \\
1.37 \%\end{array}$ & $\begin{array}{l}-5.228 \times 10^{-2} \\
3.87 \%\end{array}$ & $-5.033 \times 10^{-2}$ & $4.17 \%$ & \\
\hline $\mathbf{N}$ & Value $\delta(\% \bar{S})$ & $\begin{array}{l}2.512 \times 10^{-2} \\
2.77 \%\end{array}$ & $\begin{array}{l}2.154 \times 10^{-2} \\
11.87 \%\end{array}$ & $\begin{array}{l}2.426 \times 10^{-2} \\
0.74 \%\end{array}$ & $\begin{array}{l}2.685 \times 10^{-2} \\
9.84 \%\end{array}$ & $2.444 \times 10^{-2}$ & $9.06 \%$ & \\
\hline
\end{tabular}


between perpendiculars, and $T$ the draught. The reliability of the CFD data is assessed by the estimation of

(1) the average among all the submissions

$$
\bar{S}=\frac{1}{n} \sum_{i=1}^{n} S_{i}
$$

where $n$ is the number of the CFD data and $S_{i}$ is the value of the $i$ th submission;

(2) the standard deviation from the mean value (in percentage of the mean value)

$$
\sigma(\% \bar{S})=\frac{\sqrt{\frac{\sum_{i=1}^{n}\left(S_{i}-\bar{S}\right)^{2}}{n-1}}}{\bar{S}} \times 100
$$

(3) and, for each simulation, the deviation from the mean (as percentage of the mean value)

$$
\delta_{i}(\% \bar{S})=\frac{S_{i}-\bar{S}}{\bar{S}} \%
$$

3.3 Computational Fluid Dynamics Comparison. Hydrodynamic loads predicted by the CFD simulations for the operative conditions considered are reported in Tables 7-11; in the tables, for each hydrodynamic load component, the mean values $(\bar{S})$ between the simulations, the standard deviations from the mean $(\sigma)$ and, for each submission, its deviation from the mean $(\delta)$ (in percentage of the mean value) are also reported. Looking at the standard deviations and, for each submission, at the deviations from the mean, it can be observed that the agreement between CFD submissions is fairly good, with most of the differences in the limit of the numerical uncertainty. In particular, standard deviations are generally well below $10 \%$ among all the hydrodynamic loads and the operative conditions.

Standard deviation for $X$ is always small (less than $4 \%$ ) without any clear dependence on the speed or the drift angle; the only exception is the $\mathrm{Fr}=0.5-\beta=6 \mathrm{deg}$ test case for which a standard deviation of about $8 \%$ is reported. However, for this case, the large scatter is due to relatively small number of submission and to the large underprediction from IIHR-EARSM-GR and the large overprediction provided by Xnavis-SA. For the former, the large deviations are clearly due to lack in the grid resolution (this computation has been done on a $13 \mathrm{M}$ grid, whereas all the other simulations from IIHR have been performed on much refined grids), whereas for Xnavis-SA, the inaccuracy should be mostly due to the adopted turbulence model, the SpalartAllmaras, which is known to be rather dissipative. If $\mathrm{Fr}=0.5-\beta=6 \mathrm{deg}$ case is excluded from the mean, the averaged standard deviation for the resistance prediction would be around $3.32 \%$, i.e., a value close to what was observed at latest CFD workshops (for example, at the Gotheborg 2010 workshop for the DTMB5415, standard deviation for the resistance prediction was 3\%). Looking at the deviation from the mean for each estimation, it can be seen that, in general, computations using DES or anisotropic turbulence model provide resistance predictions in better agreement (i.e., closer to mean values) than simpler turbulence models; in particular, computations that make use of the one equation Spalart-Allmaras model generally overpredict the longitudinal force $(X)$.

Standard deviations for the lateral force $(Y)$ predictions are also rather small (generally well below 5\%). Higher scattered predictions for $Y$ are observed for $\mathrm{Fr}=0.4-\beta=6 \mathrm{deg}$ conditions $(\sigma \sim 7 \%)$; all the simulations, except for ISIS-EARSM-AMR, provide values more than $5 \%$ greater or smaller than the mean values. For what yaw moment prediction concerns, from the tables, it

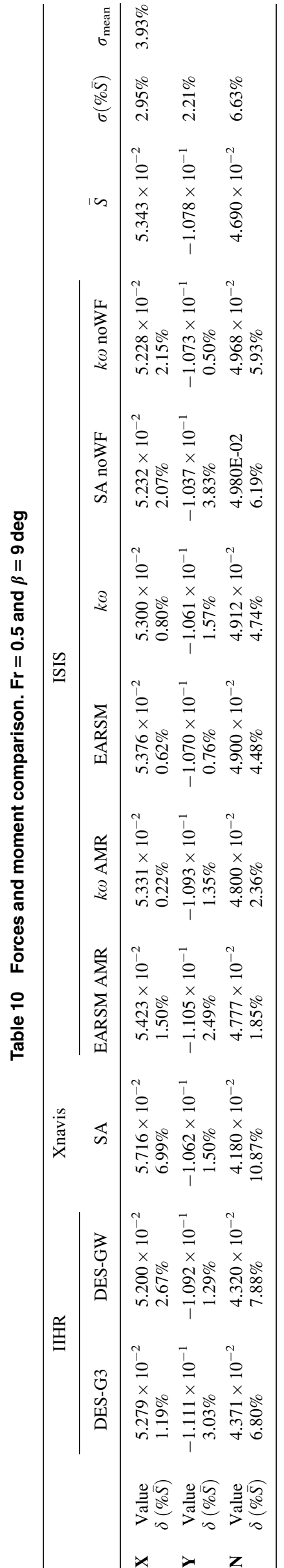


Table 11 Forces and moment comparison. $\mathrm{Fr}=0.5$ and $\beta=6 \mathrm{deg}$ (Even Keel)

\begin{tabular}{|c|c|c|c|c|c|c|c|c|}
\hline & & \multicolumn{3}{|c|}{ IIHR } & \multirow{2}{*}{$\begin{array}{c}\text { Xnavis } \\
\text { SA }\end{array}$} & \multirow[b]{2}{*}{$\bar{S}$} & \multirow[b]{2}{*}{$\sigma(\% \bar{S})$} & \multirow[b]{2}{*}{$\sigma_{\text {mean }}$} \\
\hline & & EARSM-GR & DES-G2 & DES-G3 & & & & \\
\hline $\mathrm{X}$ & $\begin{array}{l}\text { Value } \\
\delta(\% \bar{S})\end{array}$ & $\begin{array}{l}3.087 \times 10^{-2} \\
4.14 \%\end{array}$ & $\begin{array}{l}3.094 \times 10^{-2} \\
3.92 \%\end{array}$ & $\begin{array}{l}3.256 \times 10^{-2} \\
1.10 \%\end{array}$ & $\begin{array}{l}3.445 \times 10^{-2} \\
6.96 \%\end{array}$ & $3.221 \times 10^{-2}$ & $5.24 \%$ & $3.20 \%$ \\
\hline $\mathrm{Y}$ & $\begin{array}{l}\text { Value } \\
\delta(\% \bar{S})\end{array}$ & $\begin{array}{l}-5.025 \times 10^{-2} \\
2.07 \%\end{array}$ & $\begin{array}{l}-5.107 \times 10^{-2} \\
0.49 \%\end{array}$ & $\begin{array}{l}-5.391 \times 10^{-2} \\
5.05 \%\end{array}$ & $\begin{array}{l}-5.004 \times 10^{-2} \\
2.49 \%\end{array}$ & $-5.132 \times 10^{-2}$ & $3.48 \%$ & \\
\hline $\mathrm{N}$ & $\begin{array}{l}\text { Value } \\
\delta(\% \bar{S})\end{array}$ & $\begin{array}{l}2.550 \times 10^{-2} \\
0.49 \%\end{array}$ & $\begin{array}{l}2.555 \times 10^{-2} \\
0.69 \%\end{array}$ & $\begin{array}{l}2.540 \times 10^{-2} \\
0.11 \%\end{array}$ & $\begin{array}{l}2.505 \times 10^{-2} \\
1.29 \%\end{array}$ & $2.538 \times 10^{-2}$ & $0.89 \%$ & \\
\hline
\end{tabular}

Table 12 CFD verification assessment for CFD hydrodynamic loads

\begin{tabular}{|c|c|c|c|c|c|c|c|c|c|}
\hline ID & $\mathrm{Fr}$ & $\beta$ & DOF & Load & $R$ & Convergence & $U_{I} \% S$ & $U_{G} \% S$ & $U_{S N} \% S$ \\
\hline \multirow[t]{3}{*}{ Xnavis-SA } & \multirow[t]{3}{*}{0.4} & \multirow[t]{3}{*}{$6 \mathrm{deg}$} & \multirow[t]{12}{*}{$\mathrm{FX}_{\sigma \tau}$} & $\mathrm{X}$ & 0.14 & MC & - & $4.08 \%$ & $4.08 \%$ \\
\hline & & & & Y & 1.42 & MD & - & - & - \\
\hline & & & & $\mathrm{N}$ & 2.24 & MD & - & - & - \\
\hline \multirow[t]{3}{*}{ Xnavis-SA } & & \multirow[t]{3}{*}{$9 \mathrm{deg}$} & & $X$ & 0.13 & MC & - & $3.24 \%$ & $3.24 \%$ \\
\hline & & & & Y & -0.06 & $\mathrm{OC}$ & - & - & - \\
\hline & & & & $\mathrm{N}$ & 0.64 & MC & - & $9.50 \%$ & $9.50 \%$ \\
\hline \multirow{3}{*}{ Xnavis-SA } & \multirow{3}{*}{0.5} & \multirow{3}{*}{$6 \mathrm{deg}$} & & $X$ & 0.04 & MC & - & $0.57 \%$ & $0.57 \%$ \\
\hline & & & & Y & 0.39 & $\mathrm{MC}$ & - & $2.22 \%$ & $2.22 \%$ \\
\hline & & & & $\mathrm{N}$ & -0.24 & $\mathrm{OC}$ & - & - & - \\
\hline \multirow{3}{*}{ Xnavis-SA } & & \multirow[t]{3}{*}{$9 \mathrm{deg}$} & & $X$ & 0.04 & MC & - & $0.32 \%$ & $0.32 \%$ \\
\hline & & & & Y & 0.47 & MC & - & $2.59 \%$ & $2.59 \%$ \\
\hline & & & & $\mathrm{N}$ & 0.27 & MC & - & $0.23 \%$ & $0.23 \%$ \\
\hline \multirow[t]{3}{*}{ Xnavis-SA } & & \multirow[t]{3}{*}{$6 \mathrm{deg}$} & \multirow[t]{3}{*}{$\mathrm{FX}_{0}$} & X & 0.10 & MC & - & $2.22 \%$ & $2.22 \%$ \\
\hline & & & & $\mathrm{Y}$ & 2.83 & MD & - & - & - \\
\hline & & & & $\mathrm{N}$ & 2.61 & MD & - & - & - \\
\hline \multirow[t]{3}{*}{ IIHR-SST-DES-G2 } & & \multirow[t]{3}{*}{$6 \mathrm{deg}$} & & $X$ & 6.30 & MD & - & - & $\ldots$ \\
\hline & & & & $\mathrm{Y}$ & -1.90 & OD & - & - & - \\
\hline & & & & $\mathrm{N}$ & 0.29 & $\mathrm{MC}$ & - & $11.73 \%$ & $11.73 \%$ \\
\hline \multirow[t]{3}{*}{ IIHR-SST-DES-G3 } & & \multirow[t]{3}{*}{$6 \mathrm{deg}$} & & $X$ & -0.35 & $\mathrm{OC}$ & - & - & - \\
\hline & & & & $\mathrm{Y}$ & -0.72 & $\mathrm{OC}$ & - & - & - \\
\hline & & & & $\mathrm{N}$ & 1.18 & MD & - & - & - \\
\hline
\end{tabular}

Table 13 Additional simulations from IIHR. $\mathrm{FR}_{\mathrm{z} \theta}$ stands for free to sinkage and trim

\begin{tabular}{|c|c|c|c|c|c|}
\hline Fr & $\operatorname{Re}$ & Model length & $\beta$ & DOF & Turbulence model-grid \\
\hline 0.5 & $7.15 \times 10^{6}$ & $3.0 \mathrm{~m}$ & $6 \mathrm{deg}$ & $\mathrm{FR}_{\mathrm{Z \Theta}}$ & DES-G3 \\
\hline 0.30 & $6.58 \times 10^{6}$ & $3.993 \mathrm{~m}$ & $24 \mathrm{deg}$ & & $\begin{array}{l}\text { SST-G3 } \\
\text { DES-G3 }\end{array}$ \\
\hline $0.30,0.45,0.61,0.77$ & $\begin{array}{l}6.58 \times 10^{6}, 9.88 \times 10^{6} \\
1.34 \times 10^{7}, 1.69 \times 10^{7}\end{array}$ & & $\begin{array}{c}0 \mathrm{deg}, 6 \mathrm{deg}, 10 \mathrm{deg}, \\
15 \mathrm{deg}, 20 \mathrm{deg}, 30 \mathrm{deg}\end{array}$ & & SST-GR \\
\hline
\end{tabular}

Table 14 Comparison between prescribed (Fixed) and predicted (Free) sinkage $(\sigma)$ and trim $(\tau)$ results and EFD [11]. $\mathrm{Fr}=0.5$ and $\beta=6 \mathrm{deg}$.

\begin{tabular}{cccccc}
\hline & \multicolumn{3}{c}{ IIHR-DES-G3 } & \multicolumn{2}{c}{ CNR-INM (EFD) } \\
\cline { 2 - 5 } & & Fixed & Free & $\Delta(\%$ fix) & $D$ \\
\hline$X$ & Value & $3.874 \times 10^{-2}$ & $2.890 \times 10^{-2}$ & $25.4 \%$ & N/A \\
$Y$ & Value & $-5.172 \times 10^{-2}$ & $-5.276 \times 10^{-2}$ & $2.01 \%$ & N/A \\
$N$ & Value & $2.512 \times 10^{-2}$ & $2.47 \times 10^{-2}$ & $10.55 \%$ & N/A \\
$\sigma$ & Value & - & $-5.270 \times 10^{-3}$ & - & $-6.886 \times 10^{-3}$ \\
& $E[\% \mathrm{D}]$ & & $23.47 \%$ & & \\
$\tau$ & Value & - & $1.29 \mathrm{deg}$ & - & $1.71 \mathrm{deg}$ \\
& $E[\% \mathrm{D}]$ & & $24.56 \%$ & & \\
\hline
\end{tabular}

Table 15 Forces, moment, trim, and sinkage: CFD/EFD comparison. $\mathrm{Fr}=0.3$ and $\beta=24 \mathrm{deg}$. EFD data provided by BSHC [16].

\begin{tabular}{lcccc}
\hline & & SST-G3 & DES-G3 & $D$ \\
\hline$X$ & Value E (\%D) & $2.140 \times 10^{-1}$ & $2.180 \times 10^{-1}$ & $2.220 \times 10^{-1}$ \\
& & $3.71 \%$ & $1.95 \%$ & \\
$Y$ & Value E (\%D) & $-3.550 \times 10^{-1}$ & $-3.632 \times 10^{-1}$ & $-3.886 \times 10^{-1}$ \\
& & $8.66 \%$ & $6.54 \%$ & \\
$N$ & Value E (\%D) & $1.723 \times 10^{-1}$ & $1.746 \times 10^{-1}$ & $1.074 \times 10^{-1}$ \\
& & $60.39 \%$ & $62.55 \%$ & \\
$\sigma$ & Value E (\%D) & $-5.018 \times 10^{-3}$ & $-5.040 \times 10^{-3}$ & $-5.977 \times 10^{-3}$ \\
& & $16.04 \%$ & $15.68 \%$ & \\
$\tau$ & Value E (\%D) & $0.56 \mathrm{deg}$ & $0.58 \mathrm{deg}$ & $0.85 \mathrm{deg}$ \\
& & $34.12 \%$ & $31.76 \%$ & \\
\hline
\end{tabular}


Table 16 CFD verification and validation assessments for CFD hydrodynamic loads

\begin{tabular}{lccccrrrr}
\hline Load & $R$ & Convergence & $U_{I} \% S$ & $U_{G} \% S$ & $U_{S N} \% S$ & $U_{D} \% D$ & $E(\% D)$ & $U_{V} \% D$ \\
\hline $\mathrm{X}$ & 0.91 & MC & $0.013 \%$ & $42.43 \%$ & $42.43 \%$ & $10.5 \%$ & $3.71 \%$ & $43.59 \%$ \\
$\mathrm{Y}$ & 0.54 & MC & $0.163 \%$ & $3.10 \%$ & $3.10 \%$ & $3.3 \%$ & $8.66 \%$ & $4.35 \%$ \\
$\mathrm{~N}$ & 0.03 & MC & $0.006 \%$ & $0.14 \%$ & $0.14 \%$ & $2.8 \%$ & $60.39 \%$ & $2.81 \%$ \\
$\sigma$ & 2.14 & MD & - & - & - & $2.9 \%$ & $16.04 \%$ & - \\
$\tau$ & 0.15 & MC & $0.470 \%$ & $12.25 \%$ & $12.26 \%$ & $28.5 \%$ & $34.12 \%$ & $29.62 \%$ \\
\hline
\end{tabular}

Note: SST-G3, $\mathrm{Fr}=0.3$, and $\beta=24 \mathrm{deg}$.

is clear that CFD results generally show higher $\sigma$ among the hydrodynamic loads component. The large differences between CFD predictions of $N$ (larger than those observed for $Y$ ) are clearly due to the differences in the distribution of lateral force. This is ascribable to the turbulence models adopted as well as to the grid resolutions (either global, i.e., the total discretization points, or local, i.e., grid refinement in critical regions).

For $\mathrm{Fr}=0.4-\beta=6$ deg test case, IIHR conducted a rather comprehensive comparison between different turbulence models (the EARSM and the SST) on the same grid (GO) and between
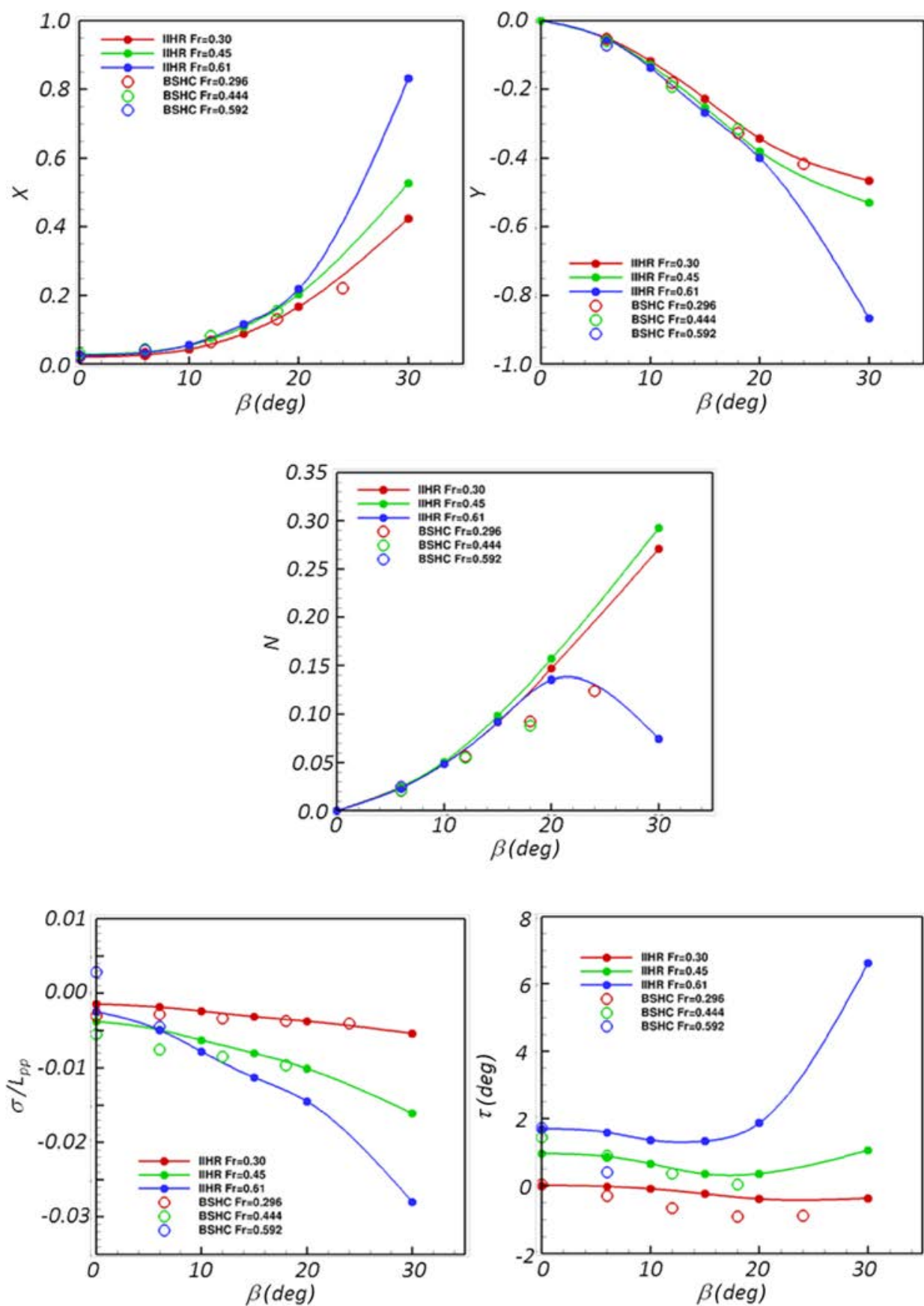

Fig. 5 Hydrodynamic loads, sinkage, and trim predictions for several Froude and drift angles. CFD data from IIHR-SST-G3, experiments from BSHC [15,24]. 
Table 17 EFD/CFD comparison, IIHR-SST-GR, in free trim and sinkage conditions; for the hydrodynamic loads, errors are reported in percentage of the data, whereas for trim and sinkage estimations are reported in percentage of the range

\begin{tabular}{|c|c|c|c|c|c|c|}
\hline \multirow[b]{2}{*}{$\mathrm{Fr}$} & \multirow[b]{2}{*}{$\beta$ (deg) } & \multicolumn{3}{|c|}{$E(\% \mathrm{D})$} & \multicolumn{2}{|c|}{$E\left(\%\left|D_{\mathrm{MAX}}-D_{\mathrm{MIN}}\right|\right)$} \\
\hline & & $\mathrm{X}$ & $\mathrm{Y}$ & $\mathrm{N}$ & $\sigma$ & $\tau$ \\
\hline \multirow[t]{6}{*}{0.296} & 0 & $26.14 \%$ & N/A & N/A & $14.98 \%$ & $5.08 \%$ \\
\hline & 6 & $16.11 \%$ & $6.92 \%$ & $22.43 \%$ & $8.75 \%$ & $7.66 \%$ \\
\hline & 12 & $10.99 \%$ & $12.67 \%$ & $15.98 \%$ & $6.69 \%$ & $16.80 \%$ \\
\hline & 18 & $0.35 \%$ & $8.76 \%$ & $32.71 \%$ & $3.40 \%$ & $21.15 \%$ \\
\hline & 24 & $11.89 \%$ & $5.08 \%$ & $68.22 \%$ & $3.28 \%$ & $1.47 \%$ \\
\hline & Average & $13.10 \%$ & $8.36 \%$ & $34.83 \%$ & $7.42 \%$ & $10.43 \%$ \\
\hline \multirow[t]{5}{*}{0.444} & 0 & $10.56 \%$ & N/A & N/A & $13.56 \%$ & $18.85 \%$ \\
\hline & 6 & $14.32 \%$ & $7.66 \%$ & $15.64 \%$ & $21.09 \%$ & $1.42 \%$ \\
\hline & 12 & $11.93 \%$ & $8.56 \%$ & $22.83 \%$ & $12.77 \%$ & $5.30 \%$ \\
\hline & 18 & $3.28 \%$ & $5.36 \%$ & $48.86 \%$ & $5.50 \%$ & $11.70 \%$ \\
\hline & Average & $10.02 \%$ & $7.20 \%$ & $29.11 \%$ & $13.23 \%$ & $9.32 \%$ \\
\hline \multirow[t]{3}{*}{0.592} & 0 & $2.03 \%$ & N/A & N/A & $43.09 \%$ & $1.78 \%$ \\
\hline & 6 & $17.41 \%$ & $26.63 \%$ & $12.06 \%$ & $2.91 \%$ & $44.81 \%$ \\
\hline & Average & $9.72 \%$ & $26.63 \%$ & $12.06 \%$ & $23.00 \%$ & $23.30 \%$ \\
\hline
\end{tabular}

Table 18 BSHC experiments: test matrix [24]

\begin{tabular}{lccc}
\hline Fr & $\mathrm{Fr}_{\mathrm{H}}$ & $\mathrm{Re}$ & $\beta(\mathrm{deg})$ \\
\hline 0.296 & 0.500 & $6.495 \times 10^{6}$ & 0 deg, 6 deg, 12 deg, 18 deg, 24 deg \\
0.444 & 0.750 & $9.742 \times 10^{6}$ & $0 \mathrm{deg}, 6 \mathrm{deg}, 12 \mathrm{deg}, 18 \mathrm{deg}$ \\
0.592 & 1.000 & $1.299 \times 10^{7}$ & 0 deg, 6 deg \\
\hline
\end{tabular}

different grid resolutions (G3 and GO) employing the same turbulence model (the EARSM). It is shown that the dependency on the turbulence model or on the grid resolution for the resistance and the yaw moment prediction is rather small (about $3 \%$ at maximum), whereas large differences are seen for the lateral force prediction (differences of $10 \%$ with the grid refinement and about $6 \%$ between the two turbulence models). An analysis of both grid

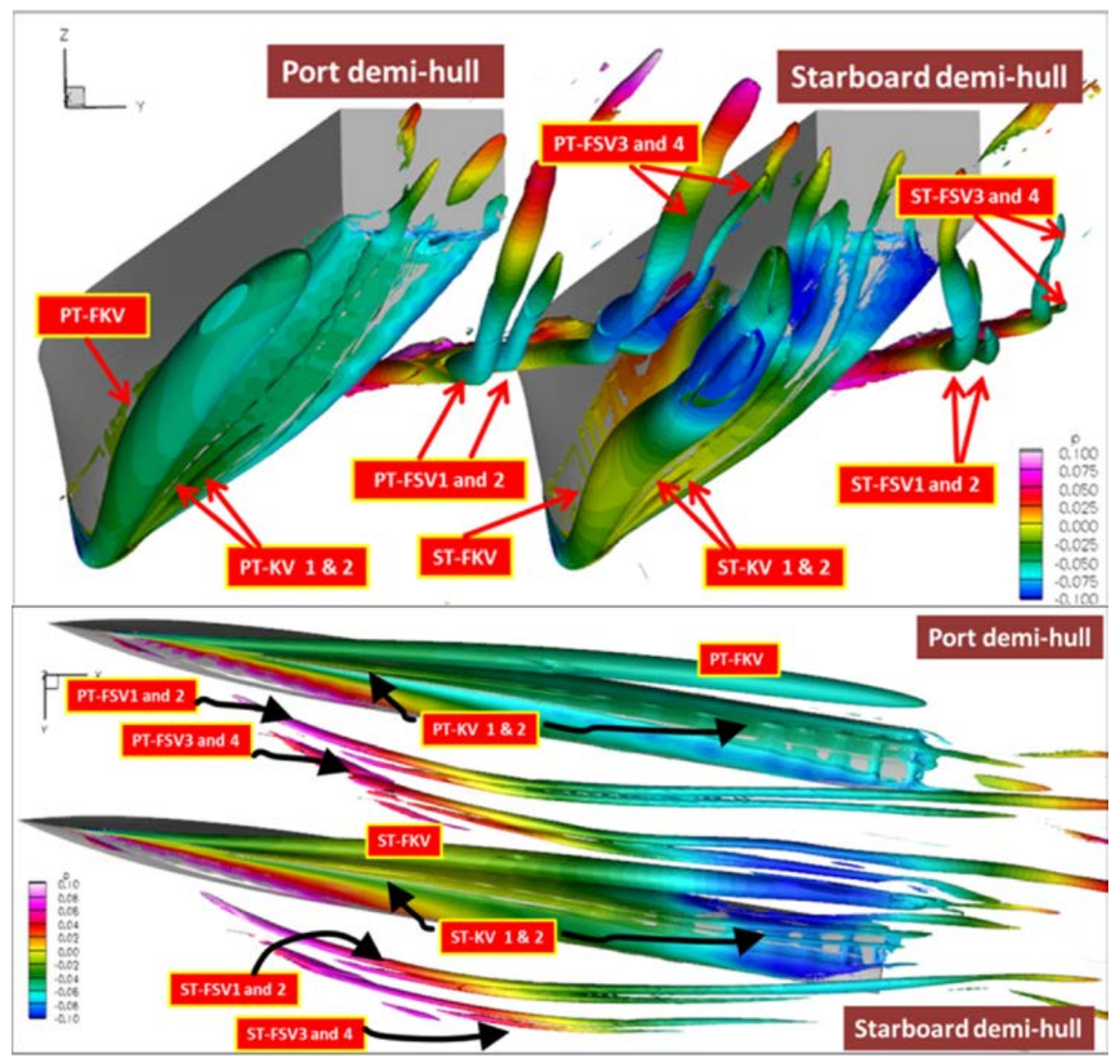

Fig. 6 Overview of vortical structures, $\mathrm{Fr}=0.5$ and $\beta=9$ deg. Xnavis-SA 10M grid. Vortical structures are identified with iso-surfaces of $\lambda_{2}=-40$ colored with nondimensional pressure. Top, perspective view; bottom, view from below. 
Table 19 S-PIV measurements: available measurement planes for different tests

\begin{tabular}{lccc}
\hline$\beta(\mathrm{deg})$ & $\mathrm{Fr}$ & $\mathrm{Re}$ & $x / L_{\mathrm{pp}}$ \\
\hline 6 & 0.40 & $5.7210^{6}$ & $0.30,0.75,0.95,1.15,1.30$ \\
& 0.50 & $7.1510^{6}$ & $0.30,0.75,0.95$ \\
& $0.50(\mathrm{EK})$ & $7.1510^{6}$ & 0.30 \\
9 & 0.40 & $5.7210^{6}$ & 030 \\
& 0.5 & $7.1510^{6}$ & 0.30 \\
\hline
\end{tabular}

strategy and turbulence model dependencies has been provided by ECN/CNRS, which performed several computations for $\mathrm{Fr}=0.5$ and $\beta=9 \mathrm{deg}$ (see Table 10); simulations have been conducted with two turbulence models (EARSM and $k \omega$ ) on two different grids: a fine grid and a coarser grid using AMR. For these simulations, a wall function $\left(y^{+} \approx 30\right)$ has been used. For the same test case, a wall-resolved grid (i.e., with $y^{+}=1$, no wall functions) has been also considered; simulations on this grid have been performed with the $k \omega$ and the Spalart-Allmaras turbulence models. From the results reported in the table, it can be observed that the difference in the results obtained with different turbulent models on the same grid is negligible (around 1\%), whereas a relatively larger dependency is seen for computations performed on different grids and the same turbulence model, with differences for $Y$ and $N$ around $3 \%$. It has to be highlighted that the results closer to the mean value (i.e., with low deviation from the mean) are obtained with the AMR approach, in particular, for the estimation of the lateral force and the yaw moment.

3.3.1 Verification Assessment. Verification assessment for the prediction of hydrodynamic loads has been provided by IIHR for DES on grids $\mathrm{G} 2$ and $\mathrm{G} 3$ for $\mathrm{Fr}=0.5$ and $\beta=6$ deg in even keel attitude and by CNR-INM for all the conditions; no verification has been provided for ISIS computations. Assessment of convergence, including numerical uncertainty, has been provided following the factor of safety method by Xing and Stern [50,51]. Results are summarized in Table 12 . In the table, $R=\varepsilon_{12} / \varepsilon_{23}$ denotes the convergence ratio, i.e., the ratio between the fine-minus the medium-grid solution $\left(\varepsilon_{12}\right)$ and the medium-minus the coarse-grid

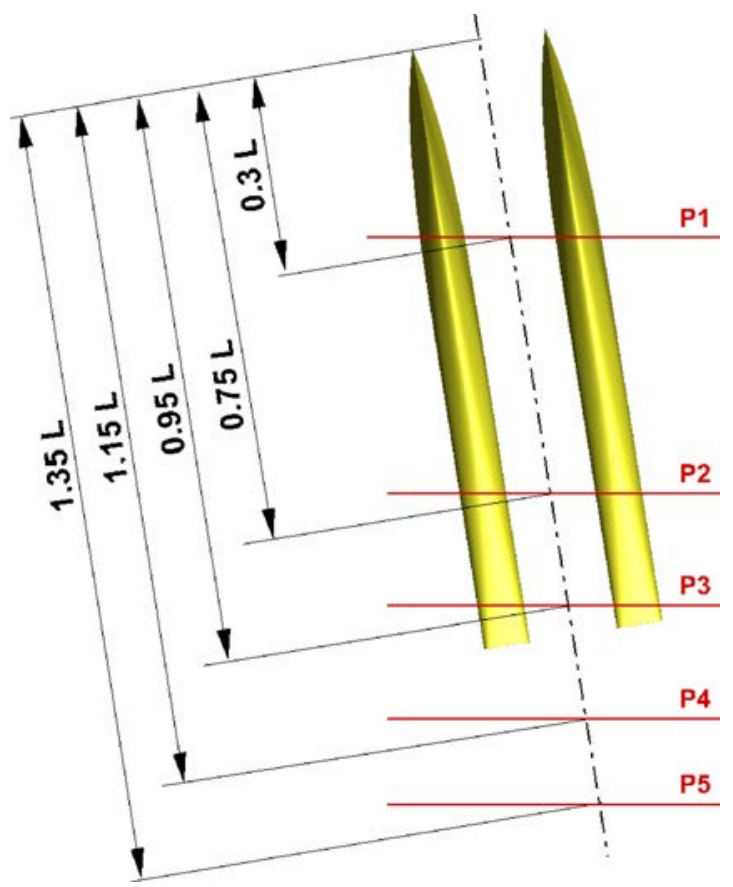

Fig. 7 S-PIV measurements: positions of the measurements planes solution $\left(\varepsilon_{23}\right)$; MC, MD, OC, and OD stand for the occurrence of monotonic convergence or divergence, and oscillatory convergence or divergence, respectively, whereas UI, UG, and USN denote iterative, grid, and simulation uncertainty, with $U_{S N}=\sqrt{U_{I}^{2}+U_{G}^{2}}$.

Computations from IIHR show monotonic convergence only for the estimation of $N$ in grid G2, whereas resistance and lateral force estimation, on this grid, show monotonic or oscillatory divergence. For computations on the triplet having G3 as the finest mesh, oscillatory convergence $(X)$ or monotonic divergence ( $Y$ and $N$ ) is observed. The bad convergence properties indicate that computations are still far from being grid independent. Most probably, this is due to the use of DES model for which LES activation region depends on the grid size. Better convergence properties and low uncertainties have been seen for Xnavis-SA computations. Monotonic or oscillatory divergence occurs for $Y$ or $N$ estimations, which are pressure dominated and strongly dependent on flow separation. At lower Fr, higher uncertainty is observed, indicating possible lack on the grid resolution around the free surface. At fixed speed, uncertainty increases with the drift angle, denoting a rather large dependency of both the separation/reattachment points and the length of the separation regions on the grid resolution. Due to the lack in the experimental data, validation, in principle, is not possible; as already noted, an attempt has been done with the interpolated data from Ref. [15]. However, since the validation assessment was not rigorous, it is not reported here; the reader is referred to Ref. [11].

3.3.2 Additional Computational Fluid Dynamics Computations. IIHR performed some additional simulations in free sinkage and trim conditions, which are summarized in Table 13, they include $\mathrm{Fr}=0.5-\beta=6 \mathrm{deg}$ for $3 \mathrm{~m}$ model length, RANS simulations for a wide range of speed $(\mathrm{Fr}=0.30-0.77)$, and drift angles ( $0 \mathrm{deg}, 6 \mathrm{deg}, 10 \mathrm{deg}, 15 \mathrm{deg}, 20 \mathrm{deg}$, and $30 \mathrm{deg}$ ) for a longer model $\left(L_{p p}=3.993 \mathrm{~m}\right.$, i.e., the model tested at BSHC [16]); and, for the same longer model, both RANS and DES simulations at large drift angle $(24 \mathrm{deg})$ and low speed $(\mathrm{Fr}=0.30)$.

In Table 14 , the results for $\mathrm{Fr}=0.5-\beta=6 \mathrm{deg}$ in free sinkage and trim conditions are reported; for completeness, the results obtained at fixed sinkage and trim using same grid and turbulence model are reported as well. By comparing the simulations at free sinkage and trim with the one conducted fixing the attitude (at the EFD position), it can be observed that a different attitude has a large influence on the resistance and the yaw moment (with a decrease of about $25 \%$ and $10 \%$, respectively, freeing the motions), whereas the influence on the lateral force is negligible (about 2\%). The difference in the resistance is mainly due to the variation of the wetted surface (which influences the frictional component of the resistance). For the yaw moment, the difference is ascribable to the difference in the lateral force distribution, indicating that the variation in the attitude leads to a variation of the position of the separation/reattachment points. Comparison with EFD data for sinkage and trim was done against CNR-INM experiments [11]. Rather large errors in the prediction of both sinkage and trim are observed (around 24\%). As already observed, no force measurements are available for this model.

Hydrodynamic loads' predictions at low speed/large drift angle ( $\mathrm{Fr}=0.3, \beta=24 \mathrm{deg})$ are summarized in Table 15. Simulations are pursued in grid G3 (44M) with DES and SST turbulence models. These simulations have been conducted for the $3.993 \mathrm{~m}$ model, i.e., considering the same model length adopted in the BSHC tests, therefore, comparison with EFD data could be done. A rather large error is observed for both the sinkage (about 16\%) and trim (about 35\%) predictions, regardless of both turbulence models adopted. As a consequence, a large discrepancy is also seen for $\mathrm{N}$ (about 60\%).

A better agreement is reported for the resistance and the lateral force predictions (error less than $4 \%$ and $9 \%$, respectively). Similar predictions are observed for the two turbulence models. Verification assessment has been provided only for RANS computation 
(Table 16). Monotonic convergence is achieved for the estimation of all the integral variables but the sinkage which shows a monotonic divergence. Numerical uncertainties are rather good for lateral force, yaw moment, and trim prediction, whereas large uncertainty is reported for the longitudinal force $(\mathrm{X})$. It has been noted $[38,39]$ that a completely different flow field has been observed on the three grids composing the triplet; steady flow was predicted on coarser grids (i.e., G4 and G5) and unsteady flow was observed on G3, which can be the reason for the large uncertainty observed for the resistance.

No uncertainty estimation is available for EFD data, however, in order to give a rough estimation, uncertainty for the DMTB 5415 in steady 10 deg drift at $\mathrm{Fr}=0.28$ is taken as Ref. [52]. The uncertainty validation $U_{v}=\sqrt{U_{S N}^{2}+U_{D}^{2}}$ is reported in Table 16 . Clearly, due to the very large comparison errors, validation is not achieved for any hydrodynamic load, a part or for the resistance, for which validation is achieved at a rather high level of validation uncertainty.

The numerical analysis conducted for various speeds/drift angles is summarized in Fig. 5 and in Table 17, where, for the sake of clearness, experimental and numerical results are reported only for those series for which tests and simulations have been conducted at similar Froude numbers. BSHC conducted several tests at different speeds and drift angles [15,24]; in Table 18, only those conditions at which computations have been performed are reported. In the table, the Froude number based on the water depth has been also reported, with the experiments being performed at a finite water depth $(\mathrm{H}=1.4 \mathrm{~m})$. Since CFD simulations and EFD tests have been conducted for different conditions ( $\mathrm{Fr}$ and $\beta$ ), a direct estimation of the comparison error was not possible. However, since the differences between EFD/CFD in the Froude numbers are relatively small, the estimations at the EFD conditions have been obtained interpolating the numerical results in the $\mathrm{Fr} / \beta$ plane by using a polyharmonic spline of second-order.

Large errors are observed, anyhow it has to be taken into account that the simulations have been pursued in free trim and sinkage conditions, and at large range of drift angle; this makes the computations rather challenging. In general, the agreement is worst for the yaw moment and for the attitude predictions. In particular, for the lower Froude case, the trends for both the resistance and the lateral force predictions are captured rather accurately; errors with respect to EFD data are rather low (generally lower for $Y$ ), with the largest discrepancy (about 26\%) for $X$ estimation at zero drift angle. Average values for the errors are reasonably good, being about $13 \%$ and $8 \%$ for $X$ and $Y$,

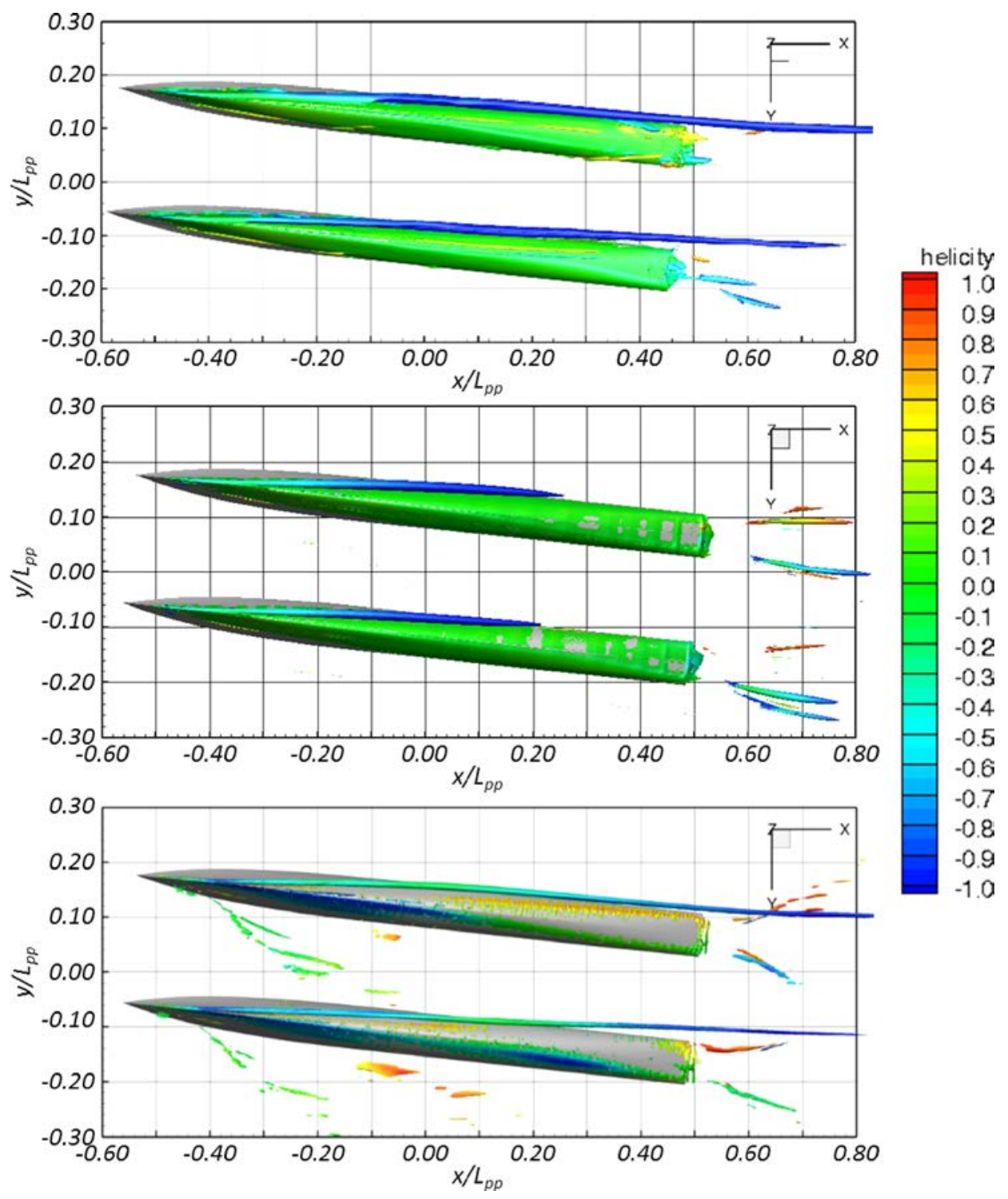

Fig. 8 Iso-surfaces of $Q=100$ colored with helicity. $F r=0.4$ and $\beta=6$ deg. View from below the free surface. Top: IIHR-G0-EARSM; middle: Xnavis-SA; bottom: ISIS-AMR-EARSM. 
respectively. Large discrepancies (average about 35\%) are observed for the yaw moment, with the error increasing with the drift angle (max about $68 \%$ ). Errors for the sinkage and trim predictions are rather large (if measured in percentage of the data); however, it has to be noted that both sinkage and trim variations are rather low and no numerical neither experimental uncertainties have been provided. Similar observation can be made for the medium speed, whereas, for the highest speed, a reduction of the error for both the resistance and the yaw moment estimations is seen. However, it has to be noted that in the latter case, only small drift angles have been tested. For an in-depth analysis of the additional tests cases, the reader is referred to Refs. [38] and [39].

\section{Vortical Structures}

An overview of the vortical structures around the catamaran in steady drift is shown in Fig. 6. Vortices are generated from both hulls as well as from breaking wave phenomena on the free surface. The main characteristics (onset, progression, and interaction with others vortices) of these structures are discussed in the following sections. The discussion is based on the CFD results for $\mathrm{Fr}=0.4-\beta=6 \mathrm{deg}$ case, being the condition for which the most stereo-particle image velocimetry (S-PIV) data are available.

4.1 Available Experimental Data. Flow measurements were taken in the "umberto pugliese" towing tank at CNR-INM. The facility is $470 \mathrm{~m}$ long, $13.5 \mathrm{~m}$ wide, and $6.5 \mathrm{~m}$ deep. The facility is equipped with a towing carriage capable of maximum speed of
$15 \mathrm{~m} / \mathrm{s}$, with fluctuations less than $0.3 \%$. Flow maps have been acquired using an in-house S-PIV system. Measurements were acquired fixing the attitude of the catamaran at the sinkage and trim values provided by the steady drift tests carried out in the same institute. The test matrix and planes of measurements are summarized in Table 19. Velocity measurements are taken in the planes orthogonal to the direction of advancement. For the sake of completeness, a brief description of the experimental setup and a summary of the results are given here; more details can be found in Ref. [25].

Stereo-particle image velocimetry data consist of (twodimensional-three components) flow survey taken on five cross planes; the position of the planes spans from the bow to the wake of the catamaran (see Fig. 7 and Table 19). It has to highlight that the first cross plane (P1) is placed at $0.2 \mathrm{Lpp}$ from the forward perpendicular, i.e., as it will be shown in the following, rather far from the onset of the main fore body keel vortices. Therefore, no direct comparison between EFD and computations will be possible for the onset of these vortices. Moreover, for higher speeds and/or larger drift angles, measurements on downstream planes could not be made; in these situations, the strong wave breaking phenomenon experienced by the bow wave on the windward sides of each hull causes air entrapment in the vortex core (ventilations), avoiding the possibility to take measurements without the risk to destroy the sensors of the cameras.

Experiments were designed to accurately acquire (three-dimensional) velocity fields on cross planes, targeting to the estimation of the following quantities:

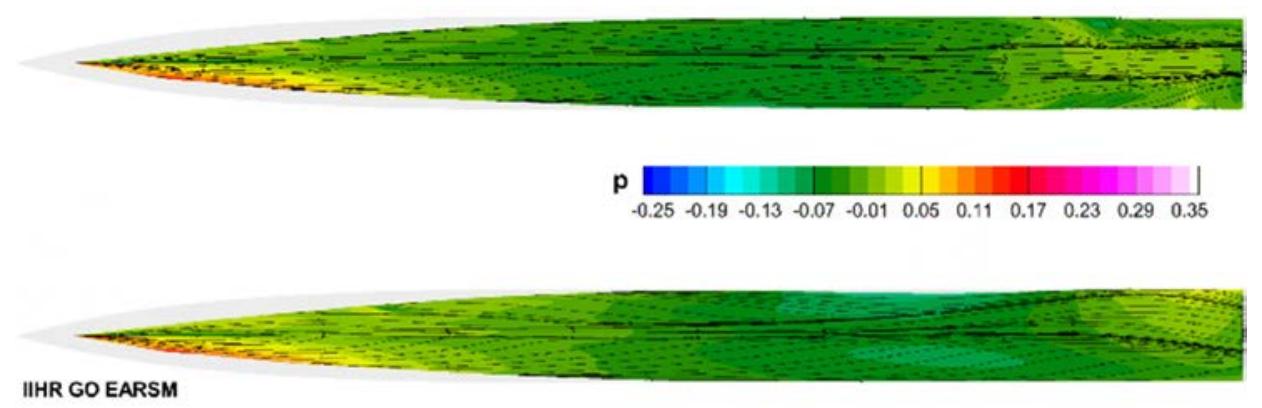

IIHR GO EARSM
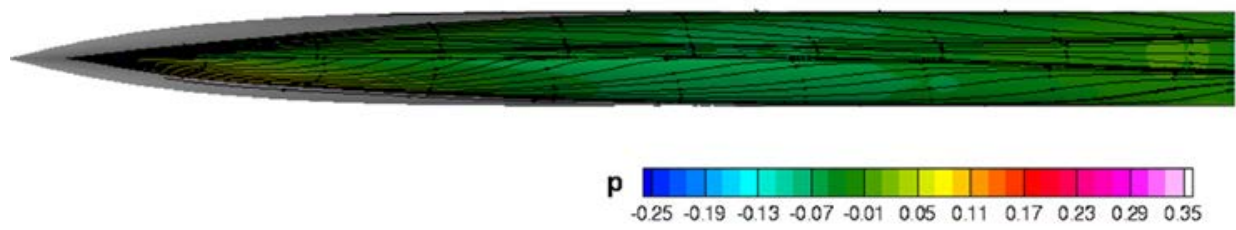

$\begin{array}{lllllllllllllll}-0.25 & -0.19 & -0.13 & -0.07 & -0.01 & 0.05 & 0.11 & 0.17 & 0.23 & 0.29 & 0.35\end{array}$

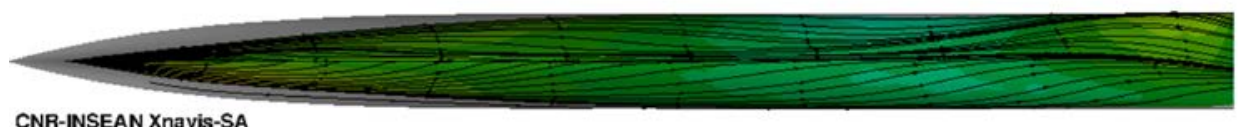

CNR-INSEAN Xnavis-SA

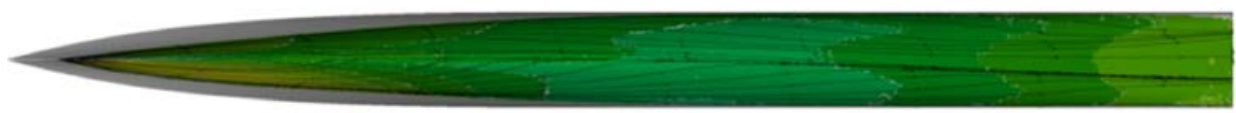

p

$\begin{array}{lllllllllll}0.25 & -0.19 & -0.13 & -0.07 & -0.01 & 0.05 & 0.11 & 0.17 & 0.23 & 0.29 & 0.35\end{array}$

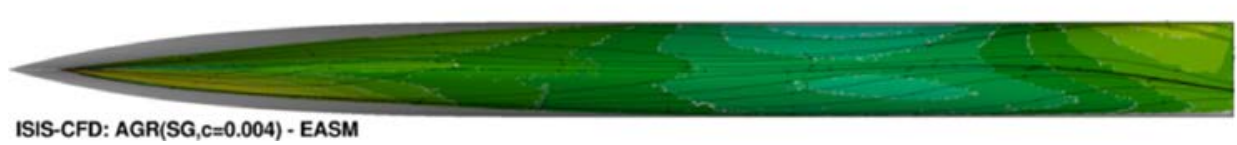

ISIS-CFD: AGR(SG,C=0.004) - EASM

Fig. 9 Limiting streamlines and surface pressure. $\mathrm{Fr}=0.4$ and $\beta=6$ deg. Bottom view. 
- mean axial velocity and mean axial vorticity;

- mean in-plane velocity field;

- RMS of in-plane and out-of-plane velocity fluctuations (i.e., all six components of the Reynolds stress tensor); and

- RMS of axial-vorticity fluctuations.

An in-depth assessment for both convergence and uncertainty has been pursued; for the sake of compactness, only main results are reported here; the full analysis can be found in Ref. [25]. For the statistical convergence analysis, a population of 500 fields was considered; two points (one in the vortex core and the other in the undisturbed flow region) have been investigated. The assessment, for the point in the vortex core, provides percentage errors for the first-order statistics within $1 \%$ of the undisturbed velocity, around $10 \%$ for the second-order statistical estimators of the velocity and to $20 \%$ for the turbulent kinetic energy. For derived quantities (such as vorticity and circulation), convergence errors are up to $1 \%$ and less than $20 \%$ for the first- and second-order estimators, respectively. A significant reduction of the errors has been

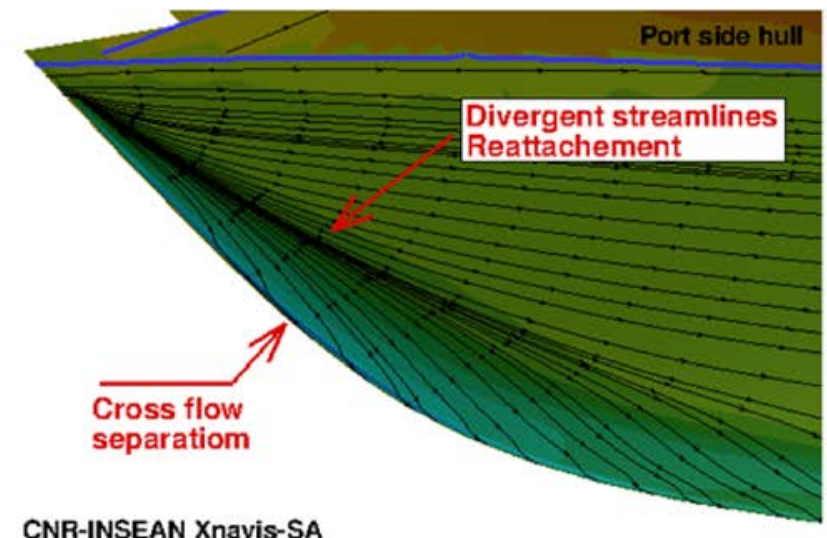

CNR-INSEAN Xnavis-SA

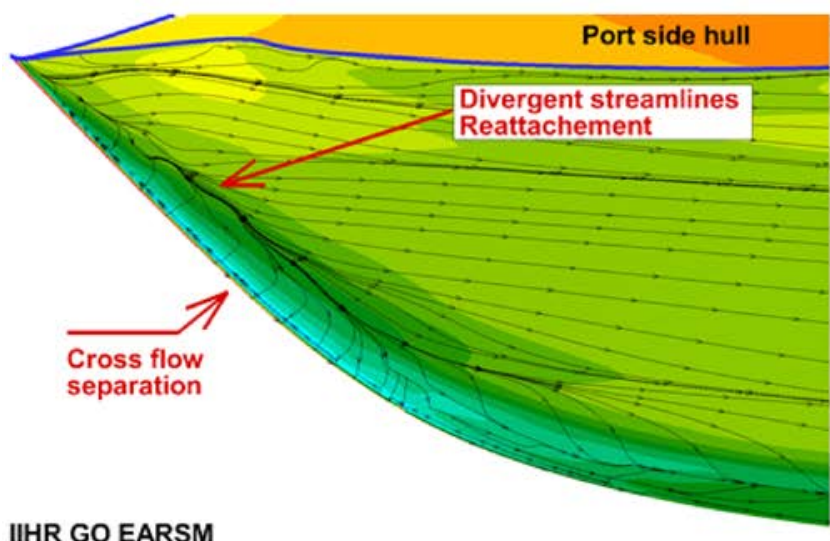

IIHR GO EARSM

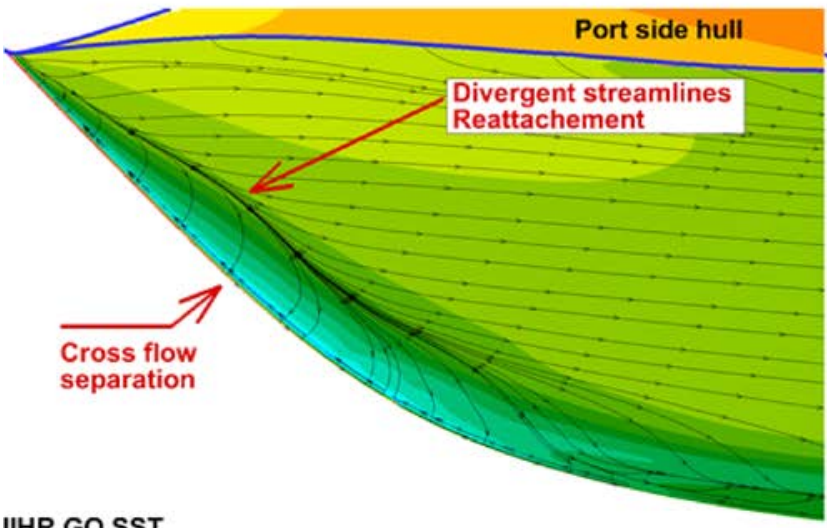

IIHR GO SST observed for the statistical estimators in the undisturbed flow region: to summarize, $0.6 \%$ and $3 \%$ at most for the first- and second-order estimators, $7 \%$ for the TKE.

Uncertainty assessment has been pursued following the ASME PTC 19.1-2005 procedure [53] and the approach proposed in Ref. [52]; uncertainty for the keel vortex (KV) parameters was based on the analysis of the instantaneous traces of the leeward vortex. The levels of random and systematic uncertainties for the vortex core parameters were around $0.53 \%$ and $1.03 \%$ for the mean velocities and about $2 \%$ and $0.6 \%$ for TKE, respectively. For the corresponding expanded uncertainty values, around $2.25 \%$ for the mean velocity components and $4.6 \%$ for TKE have been estimated. Uncertainty values reduce significantly in the undisturbed flow region.

4.2 Method of Analysis. The analysis of the vortices shed from the hulls of the catamaran, including their onset and progression, is carried out following the approaches described here. Their
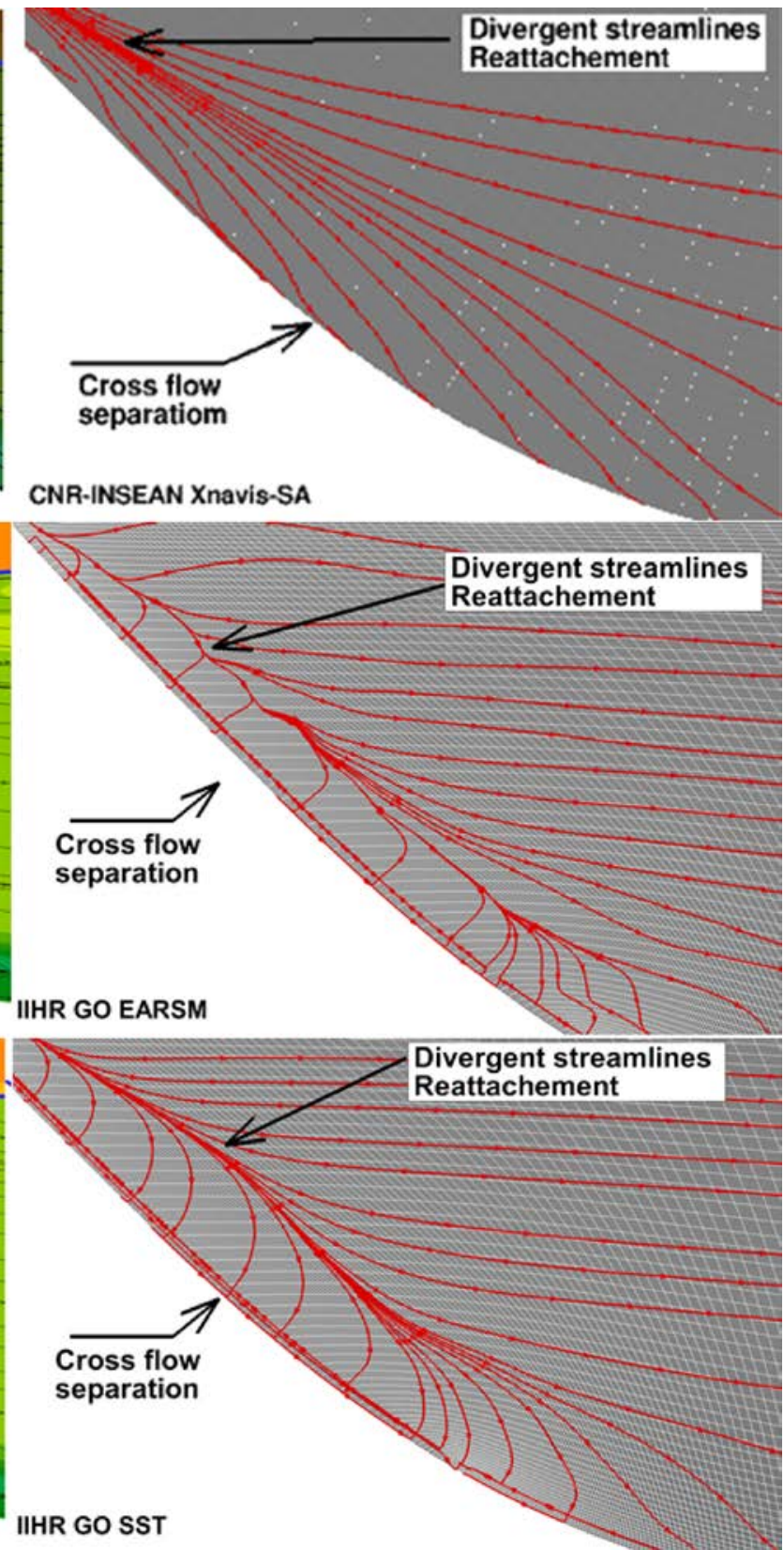

Fig. 10 Close-up view of the limiting streamlines and surface pressure (left) and surface grid (right), only port side. Fr $=0.4$ and $\beta=6$ deg (Xnavis and CFDship-IOWA results). 
analysis is made by means of the CFD solutions, since S-PIV measurements are available only in a limited number of planes [25].

In the following, coherent structures are identified as isosurfaces of either $\mathbf{Q}$-factor

$$
\mathbf{Q}=\frac{1}{2}\left[\left\|\mathbf{\Omega}^{2}\right\|-\left\|\mathbf{S}^{2}\right\|\right]
$$

or negative values of the second greater eigenvalues $\left(\lambda_{2}\right)$ of the $\left\|S_{i j}^{2}\right\|+\left\|\Omega_{i j}^{2}\right\|$ tensor, where $S_{i j}$ and $\Omega_{i j}$ are, respectively, the symmetric and the anti-symmetric components of $\mathbf{\nabla} \mathbf{U}$, i.e.,

$$
\begin{aligned}
& \mathbf{S}=\frac{1}{2}\left[\boldsymbol{\nabla} \mathbf{U}+\nabla \mathbf{U}^{\mathrm{T}}\right] \\
& \mathbf{\Omega}=\frac{1}{2}\left[\boldsymbol{\nabla} \mathbf{U}-\nabla \mathbf{U}^{\mathrm{T}}\right]
\end{aligned}
$$

As it will be shown in the following, both numerical simulations and experimental data provide steady vortex pattern; therefore, the investigation of the coherent structures is based on the steady separation and reattachment analysis [54,55]. Assessment of the CFD predictions is also made for the progression of the main vortices; the comparison between CFD and against EFD is provided for flow variables in the vortex core such as the axial vorticity, the velocity, the turbulent kinetic energy, and the location of the vortex core itself. The vortex core is identified as the location where the peak of the axial vorticity $\left(\omega_{x}\right)$ occurs: $\omega_{x}$ at vortex core is also used as an estimation of the vortex strength. This analysis allows an in-depth study of the grid resolution and turbulence models effects on flow predictions, as well as to validate the CFD predictions for the onset and vortex progression.

\subsection{Computational Fluid Dynamics Comparison}

4.3.1 Fore Body Keel Vortices. The steady drift advancement causes 3D flow separations and the formation of two strong vortices along the two hulls (Fig. 6); these long lived structures, which result to be more intense, will be denoted as port side fore body keel vortices (PT-FKV) and starboard side fore body keel vortices (ST-FKV), respectively. In Figs. 8 and 9, best results from each group are shown; Fig. 8 highlights the vortical structures using Qcriteria, whereas Fig. 9 reports limiting streamlines along the hull surfaces. In general, all the submissions are capable to capture the formation and the dynamics of these vortical structures, with the main differences residing in the intensity and dissipation rate. Clearly, simulations using more sophisticated turbulence models (in principle the anisotropic ones, either $\kappa \omega$-SST or EARSM) provide stronger and more persistent vortices than the simulations using one-equation isotropic model.

From the figures of the limiting streamlines, it is clear that PTFKV and ST-FKV are generated by the flow separation along the keel caused by the freestream crossflow. Due to the sharp bow edge of the hulls, the onsets of these vortices are located at the forward perpendiculars of each demi-hull; going downstream, the crossflow separation along the keel continuously feeds the FKVs. As it can be seen in Figs. 6 and 8, for both PT-FKV and ST-FKV, the lateral displacement increases downstream. However, due to the straightening effect in the middle of the two hulls, the lateral displacement of PT-FKV is larger than that of ST-FKV. Both vortices move toward the free-surface; due to the interaction with the
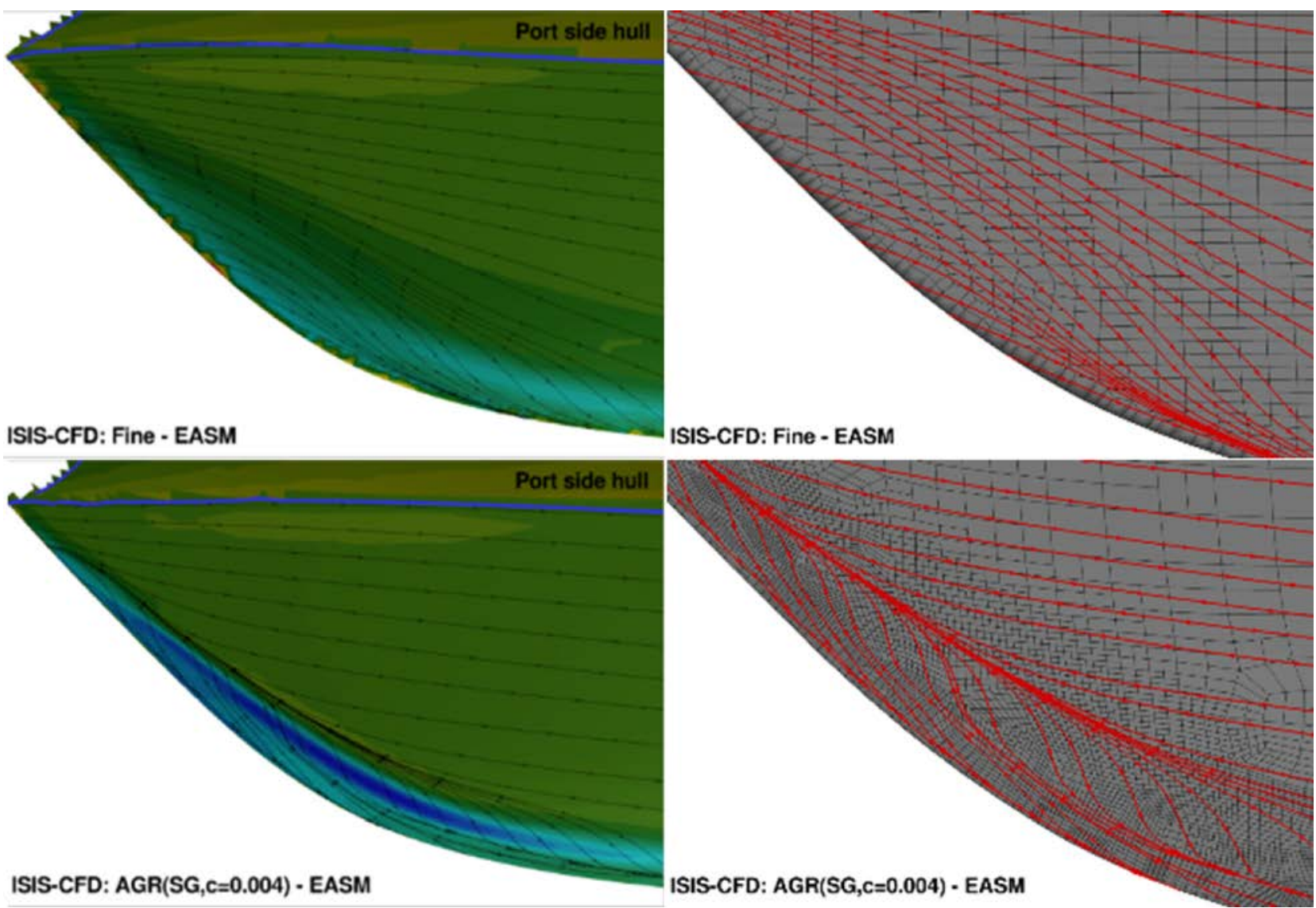

Fig. 11 Close-up view of the limiting streamlines and surface pressure (left) and surface grid (right), only port side. $\mathrm{Fr}=0.4$ and $\beta=6 \mathrm{deg}$ (ISIS results). 
free surface, the ST-FVK shows a weaker rise. Moreover, due to the presence of a deep wave trough in the inner region, the starboard side vortex shows an initial downward movement (at least for the higher speed/larger drift angle, i.e., the condition for which the inner wave is deeper).

All the submissions show similar pattern for the converging streamlines (Fig. 9), indicating similar generation for these vortices. A more in-depth analysis of the onset for these vortices can be inferred from Figs. 10 and 11, where close-up views around the bow are shown for Xnavis-SA, IIHR (SST and EARSM on GO grid), and ISIS-EARSM (with and w/o AMR). All the submissions highlight an open-type separation and reattachment, with the separation at the sharp edge and the reattachment between the free surface and the keel. Comparing results which are using different turbulence models on the same grid, a rather weak dependency on the turbulence model is seen; on the contrary, the onset phase of FKVs is strongly affected by grid resolution, with only wellrefined grids able to accurately capture the details of the flow in the onset region (see, for example, ISIS-EARSM with and without AMR).

Table 20 Axial vortex core prediction errors for PT-FKV at $\mathrm{Fr}=0.4$ and $\beta=6 \mathrm{deg}$

\begin{tabular}{|c|c|c|c|c|c|c|c|}
\hline \multirow[b]{2}{*}{ CFD } & \multirow[b]{2}{*}{$x / L_{p p}$} & \multicolumn{6}{|c|}{ Flow variable error $(\% D)$} \\
\hline & & $y / L_{p p}$ & $z / L_{p p}$ & $u$ & TKE & $\omega_{x}$ & Overall \\
\hline \multirow[t]{6}{*}{ IIHR-G3-EARSM } & 0.298 & $1.3 \%$ & $1.0 \%$ & $13.8 \%$ & $90.8 \%$ & $68.1 \%$ & $35.0 \%$ \\
\hline & 0.746 & $1.3 \%$ & $0.5 \%$ & $4.4 \%$ & $95.4 \%$ & $87.1 \%$ & $37.7 \%$ \\
\hline & 0.945 & $2.7 \%$ & $7.5 \%$ & $0.4 \%$ & $96.7 \%$ & $87.6 \%$ & $39.0 \%$ \\
\hline & 1.144 & $12.1 \%$ & $202.8 \%$ & $5.6 \%$ & $95.2 \%$ & $82.1 \%$ & $79.5 \%$ \\
\hline & 1.293 & $17.9 \%$ & $254.5 \%$ & $0.6 \%$ & $96.8 \%$ & $88.4 \%$ & $91.6 \%$ \\
\hline & Average & $7.1 \%$ & $93.3 \%$ & $4.9 \%$ & $95.0 \%$ & $82.7 \%$ & $56.6 \%$ \\
\hline \multirow[t]{6}{*}{ IIHR-G3-SST } & 0.298 & $1.3 \%$ & $1.0 \%$ & $15.8 \%$ & $93.3 \%$ & $65.9 \%$ & $22.3 \%$ \\
\hline & 0.746 & $1.3 \%$ & $0.5 \%$ & $5.1 \%$ & $96.0 \%$ & $85.5 \%$ & $37.7 \%$ \\
\hline & 0.945 & $8.6 \%$ & $7.5 \%$ & $1.8 \%$ & $96.5 \%$ & $87.6 \%$ & $40.4 \%$ \\
\hline & 1.144 & $15.8 \%$ & $169.9 \%$ & $5.8 \%$ & $95.1 \%$ & $83.4 \%$ & $74.0 \%$ \\
\hline & 1.293 & $17.9 \%$ & $254.5 \%$ & $0.2 \%$ & $96.9 \%$ & $89.4 \%$ & $91.8 \%$ \\
\hline & Average & $9.0 \%$ & $86.7 \%$ & $5.7 \%$ & $95.5 \%$ & $82.4 \%$ & $55.9 \%$ \\
\hline \multirow[t]{6}{*}{ IIHR-GO-EARSM } & 0.298 & $2.9 \%$ & $0.8 \%$ & $14.4 \%$ & $85.5 \%$ & $54.5 \%$ & $31.6 \%$ \\
\hline & 0.746 & $0.9 \%$ & $0.9 \%$ & $3.3 \%$ & $92.3 \%$ & $76.5 \%$ & $34.8 \%$ \\
\hline & 0.945 & $7.5 \%$ & $6.4 \%$ & $0.1 \%$ & $94.9 \%$ & $80.0 \%$ & $37.8 \%$ \\
\hline & 1.144 & $17.1 \%$ & $198.8 \%$ & $6.7 \%$ & $93.6 \%$ & $75.6 \%$ & $78.4 \%$ \\
\hline & 1.293 & $17.6 \%$ & $313.9 \%$ & $1.3 \%$ & $95.6 \%$ & $84.5 \%$ & $102.6 \%$ \\
\hline & Average & $9.2 \%$ & $104.2 \%$ & $5.2 \%$ & $92.4 \%$ & $74.2 \%$ & $57.0 \%$ \\
\hline \multirow[t]{6}{*}{ IIHR-GO-SST } & 0.298 & $2.9 \%$ & $1.6 \%$ & $17.6 \%$ & $89.7 \%$ & $48.9 \%$ & $32.1 \%$ \\
\hline & 0.746 & $0.9 \%$ & $0.9 \%$ & $4.8 \%$ & $92.9 \%$ & $78.9 \%$ & $35.7 \%$ \\
\hline & 0.945 & $5.7 \%$ & $5.5 \%$ & $1.9 \%$ & $95.0 \%$ & $83.4 \%$ & $38.3 \%$ \\
\hline & 1.144 & $16.2 \%$ & $191.5 \%$ & $6.6 \%$ & $92.4 \%$ & $81.2 \%$ & $77.6 \%$ \\
\hline & 1.293 & $19.6 \%$ & $260.9 \%$ & $0.0 \%$ & $95.0 \%$ & $89.1 \%$ & $92.9 \%$ \\
\hline & Average & $9.1 \%$ & $92.0 \%$ & $6.2 \%$ & $93.0 \%$ & $76.3 \%$ & $55.3 \%$ \\
\hline \multirow[t]{6}{*}{ Xnavis-SA } & 0.298 & $2.3 \%$ & $0.5 \%$ & $10.9 \%$ & $86.2 \%$ & $78.1 \%$ & $35.6 \%$ \\
\hline & 0.746 & $2.8 \%$ & $1.0 \%$ & $2.2 \%$ & $88.7 \%$ & $91.5 \%$ & $37.2 \%$ \\
\hline & 0.945 & $8.1 \%$ & $4.1 \%$ & $0.8 \%$ & $93.9 \%$ & $93.0 \%$ & $40.0 \%$ \\
\hline & 1.144 & $19.5 \%$ & $178.0 \%$ & $4.9 \%$ & $91.6 \%$ & $91.0 \%$ & $77.0 \%$ \\
\hline & 1.293 & $24.7 \%$ & $248.4 \%$ & $2.2 \%$ & $93.4 \%$ & $94.4 \%$ & $92.6 \%$ \\
\hline & Average & $11.5 \%$ & $86.4 \%$ & $4.2 \%$ & $90.7 \%$ & $89.6 \%$ & $56.5 \%$ \\
\hline \multirow[t]{6}{*}{ ISIS-EARSM } & 0.298 & $3.7 \%$ & $7.4 \%$ & $7.3 \%$ & $99.8 \%$ & $80.7 \%$ & $39.8 \%$ \\
\hline & 0.746 & $3.2 \%$ & $3.2 \%$ & $5.4 \%$ & $99.5 \%$ & $88.5 \%$ & $40.0 \%$ \\
\hline & 0.945 & $7.3 \%$ & $6.3 \%$ & $3.9 \%$ & $99.5 \%$ & $90.5 \%$ & $41.5 \%$ \\
\hline & 1.144 & $20.0 \%$ & $196.8 \%$ & $9.8 \%$ & $99.5 \%$ & $87.0 \%$ & $82.6 \%$ \\
\hline & 1.293 & $24.9 \%$ & $306.1 \%$ & $1.8 \%$ & $99.8 \%$ & $91.4 \%$ & $104.8 \%$ \\
\hline & Average & $11.8 \%$ & $104.0 \%$ & $5.6 \%$ & $99.6 \%$ & $87.6 \%$ & $61.7 \%$ \\
\hline \multirow[t]{6}{*}{ ISIS-EARSM-AMR } & 0.298 & $3.9 \%$ & $8.1 \%$ & $23.8 \%$ & $99.9 \%$ & $56.1 \%$ & $38.4 \%$ \\
\hline & 0.746 & $2.3 \%$ & $1.9 \%$ & $12.3 \%$ & $99.4 \%$ & $84.2 \%$ & $40.0 \%$ \\
\hline & 0.945 & $6.2 \%$ & $4.6 \%$ & $8.1 \%$ & $99.6 \%$ & $89.4 \%$ & $41.6 \%$ \\
\hline & 1.144 & $18.5 \%$ & $195.4 \%$ & $11.9 \%$ & $99.6 \%$ & $85.3 \%$ & $82.1 \%$ \\
\hline & 1.293 & $23.6 \%$ & $304.0 \%$ & $3.5 \%$ & $99.8 \%$ & $90.4 \%$ & $104.3 \%$ \\
\hline & Average & $10.9 \%$ & $102.8 \%$ & $11.9 \%$ & $99.6 \%$ & $81.1 \%$ & $61.3 \%$ \\
\hline \multirow[t]{6}{*}{ ISIS- $k \omega$} & 0.298 & $3.7 \%$ & $7.0 \%$ & $6.0 \%$ & $97.7 \%$ & $80.7 \%$ & $39.0 \%$ \\
\hline & 0.746 & $3.2 \%$ & $3.3 \%$ & $2.9 \%$ & $96.5 \%$ & $88.7 \%$ & $38.9 \%$ \\
\hline & 0.945 & $7.3 \%$ & $6.7 \%$ & $1.1 \%$ & $96.8 \%$ & $91.0 \%$ & $40.6 \%$ \\
\hline & 1.144 & $20.0 \%$ & $199.4 \%$ & $6.2 \%$ & $95.0 \%$ & $88.5 \%$ & $81.8 \%$ \\
\hline & 1.293 & $25.5 \%$ & $314.1 \%$ & $2.3 \%$ & $96.6 \%$ & $92.8 \%$ & $106.3 \%$ \\
\hline & Average & $11.9 \%$ & $106.1 \%$ & $3.7 \%$ & $96.5 \%$ & $88.4 \%$ & $61.3 \%$ \\
\hline \multirow[t]{6}{*}{ ISIS- $k \omega$-AMR } & 0.298 & $4.0 \%$ & $7.9 \%$ & $11.4 \%$ & $89.1 \%$ & $64.1 \%$ & $35.3 \%$ \\
\hline & 0.746 & $2.0 \%$ & $3.3 \%$ & $3.1 \%$ & $92.0 \%$ & $87.9 \%$ & $37.7 \%$ \\
\hline & 0.945 & $6.8 \%$ & $6.9 \%$ & $0.7 \%$ & $94.5 \%$ & $91.2 \%$ & $40.0 \%$ \\
\hline & 1.144 & $20.2 \%$ & $199.4 \%$ & $5.4 \%$ & $92.9 \%$ & $89.0 \%$ & $81.4 \%$ \\
\hline & 1.293 & $25.5 \%$ & $320.1 \%$ & $3.2 \%$ & $95.4 \%$ & $93.3 \%$ & $107.5 \%$ \\
\hline & Average & $11.7 \%$ & $107.5 \%$ & $4.8 \%$ & $92.8 \%$ & $85.1 \%$ & $60.4 \%$ \\
\hline
\end{tabular}


Less accurate predictions of the onset phase by Xnavis-SA and ISIS without AMR are mainly ascribable to the coarse grid resolution around the bow region. Some differences are also seen in the reattachment phase; both IIHR and ISIS-AMR provide sharper reattachment, closer to the hull edge indicating a more intense vortex than Xnavis-SA. In addition, IIHR and ISIS-AMR show a second separation and reattachment close to the main onset of the FKVs. This flow characteristic is not well captured by Xnavis-SA and ISIS (without AMR capabilities activated). Looking at the closeup views of the limiting streamlines in Figs. 10 and 11, it is clear that these differences are due to the grid resolution around the sharp edge; IIHR-GO is more refined than Xnavis-SA (in particular close to the edge) and the grid for ISIS-AMR is the finest among all submissions. Lack in grid resolution may be the cause of the weakness in capturing the second separation/reattachment by Xnavis-SA and ISIS-EARSM without AMR simulation.

4.3.1.1 Vortex core analysis. Vortex core analysis has been conducted for the port side and the starboard side fore body keel vortices (PT-FKV and ST-FKV): in Ref. [11], all Fr- $\beta$ conditions have been deeply investigated. Here, for the sake of compactness, only the analysis of PT-FKV at $\mathrm{Fr}=0.4 / \beta=6$ is reported. Comparison between CFD submissions and against EFD will be reported for the variations along the vortex core $(x)$ of position $(y, z)$ of the vortex, axial vorticity $(\omega x)$, axial velocity $(u)$, TKE, and $Q$-factor. Results are summarized in Table 20 and Fig. 12. In the table, for each plane, errors with respect to experimental values are reported in percentage of the EFD data. For comparison purposes, for each parameter and for each submission, the averaged error among the five planes and, for each plane on the last column, overall average errors (among the parameters, for each plane and for each submission) are reported as well. Comparison in Fig. 12 shows that all the submissions provide similar results for the vortex core position. As it is also shown in Table 20, differences against EFD are rather low for the first three planes $(8 \%$ maximum for both lateral and vertical positions among all the submissions); the errors increase moving downstream (up $20-25 \%$ for the lateral position, even larger for the vertical position). However, since differences between CFD are negligible (see top panels in Fig. 12), large comparison errors in the last two planes seem due to uncertainty on the EFD data (the position of the catamaran) rather than inaccuracies in the CFD predictions.
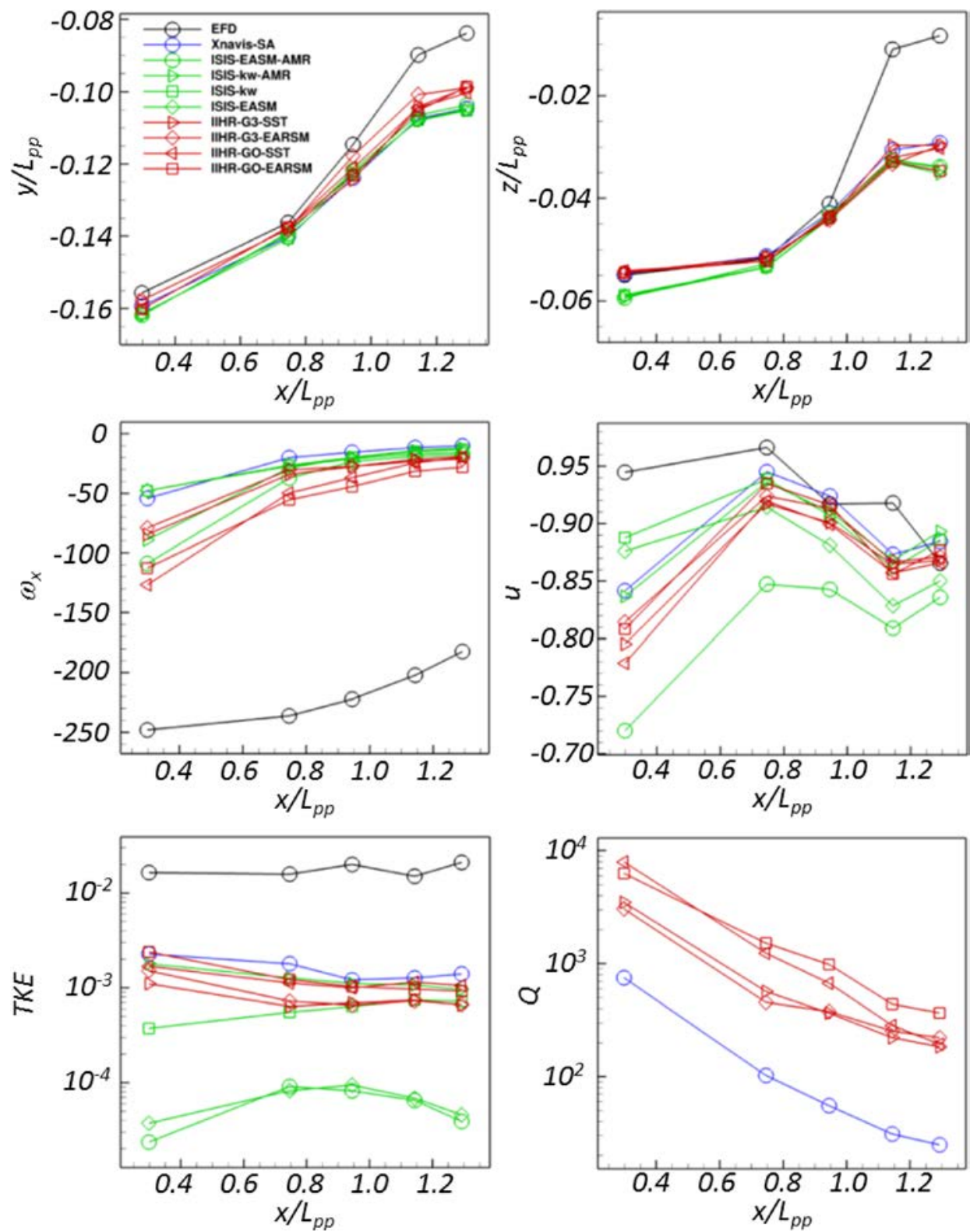

Fig. 12 Variation of vortex core parameters along $x / L_{p p}$ for PT-FKV at $F r=0.4$ and $\beta=6$ deg 
Axial vorticity variation along vortex core for PT-FKV is shown in Fig. 12; error with respect to EFD is reported in Table 20. Large differences are seen between CFD submissions; very large comparison errors are seen for all submissions against EFD data. At the first plane (i.e., $x / L_{\mathrm{pp}}=0.298$, the closest to the onset), differences between computational results are primarily due to the difference in the grid resolution; predicted axial vorticity values from most refined grids, namely ISIS-EARSM-AMR and IIHRGO (regardless of the turbulent model), are about 120-130. All the other simulations (i.e., on coarser grids) estimate values well below 100. In particular, comparing IIHR-G3-SST and IIHR-GOSST, i.e., coarse and fine grid computations adopting the same turbulence model, values at the onset plane are about 130 and 85 , respectively; local error at this plane is reduced from $65.9 \%$ (with G3) to $48.9 \%$ (with GO) (see Table 20).

Similar trend is observed for IIHR-EARSM with GO and G3 and for the ISIS-EARSM computations with and without AMR, with a decrease of the local error for $\omega_{x}$ at the onset plane from $80.7 \%$ to $56.1 \%$. These observations confirm that, at the onset, differences among submissions are mainly due to different grid

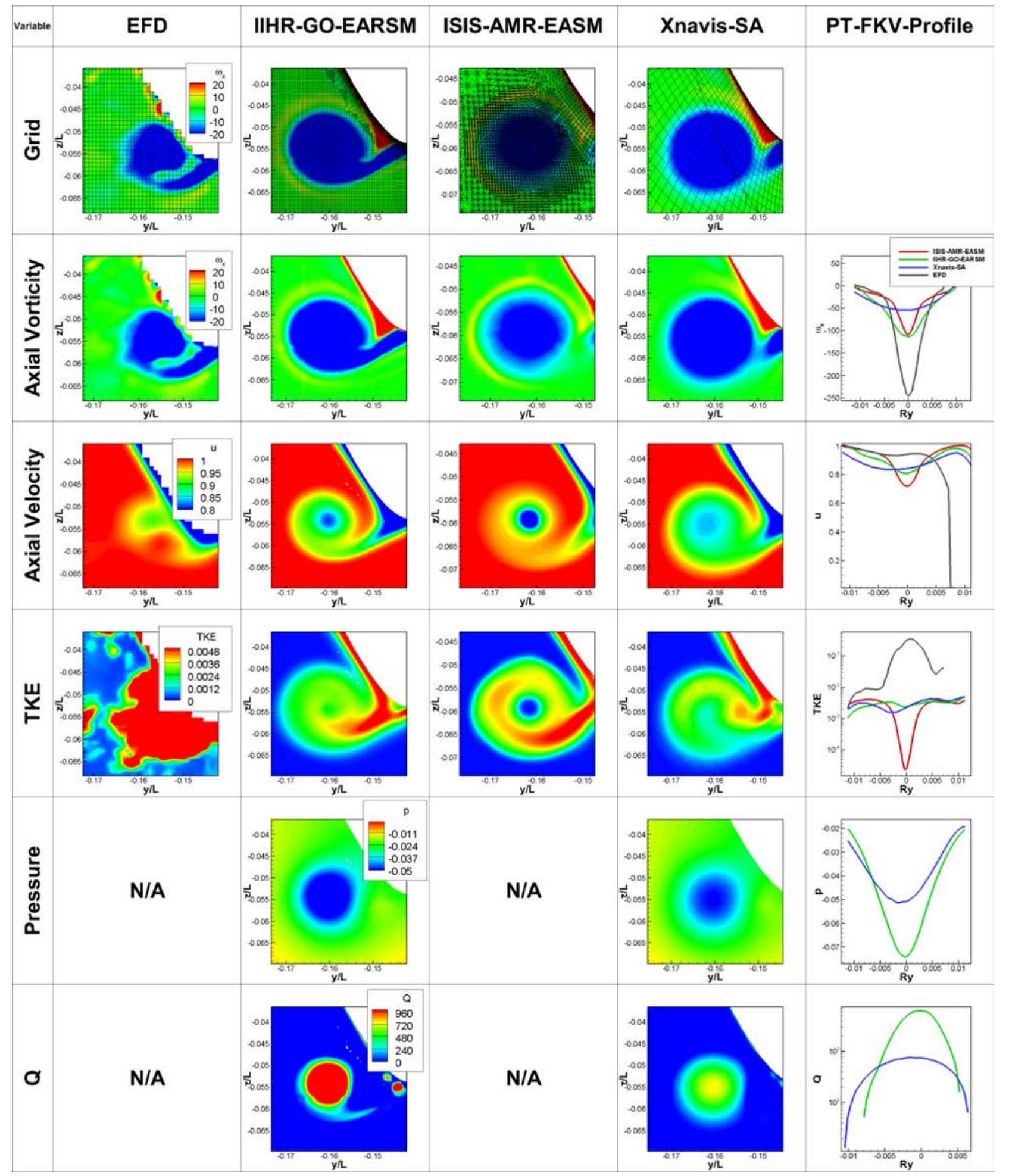

Fig. 13 Cross plane PT-FKV at P1. Fr $=0.4$ and $\beta=6$ deg. 
refinement. Moving downstream, the vorticity starts to decay; generally, the differences between CFD predictions decrease (in absolute value as distance from the mean), whereas the errors with respect to EFD increase (with comparison errors up to $90 \%$ ). Data do not allow to highlight clearly any grid or turbulence model dependency. Similar behavior is seen for the prediction of the ST-FKV (not reported here, see Ref. [11]), with errors generally lower than PT-FKV. TKE predictions are much smaller than experiments (at least one order of magnitude), with an overall error of about $90-100 \%$. Higher values are predicted by those computations performed on more refined grid (IIHR-GO and ISIS- $k \omega$-AMR). High TKE values are also predicted by XnavisSA computations. For the estimation of the longitudinal velocity $(u)$, differences between CFD are much smaller than those for $\omega_{x}$ and TKE (standard deviations is about $10 \%$ ). Comparison errors against EFD are also much lower (maximum values averaged on the five planes is about $11 \%$, rarely higher than $6 \%$, see Table 20); comparison error generally increases for larger drift angle and/or higher speeds. The error with respect to measurements decreases moving downstream, indicating inaccuracy in the prediction in the

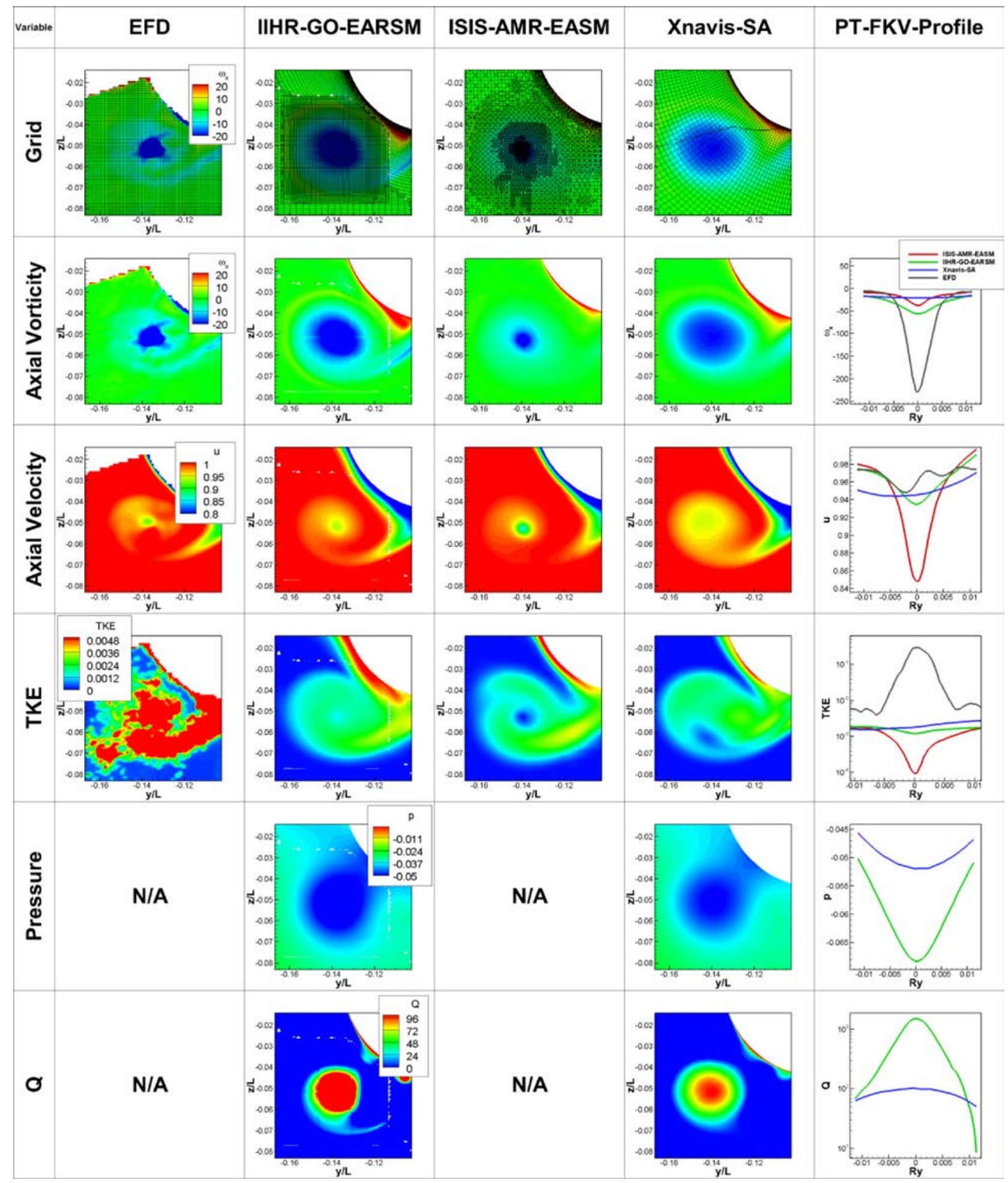

Fig. 14 Cross plane PT-FKV at P3. Fr $=0.4$ and $\beta=6$ deg. 
onset phase. No clear dependency on the grid resolution or turbulence model is seen.

4.3.1.2 Cross planes. For an in-depth assessment of CFD capabilities, comparison between estimations and against S-PIV measurements has been pursued for flow field variables on cross planes. In Figs. 13-15, closeup views of the main flow field variables (axial vorticity, axial velocity, and TKE) in the vortex core region are reported for planes P1, P3, and P5. As in Sec. 4.3.1.1, the analysis is conducted only for the port side fore body keel vortex for $\mathrm{Fr}=0.4-\beta=6 \mathrm{deg}$ condition. In the figures, only the best computations from each participating organization are reported and compared with CNR-INM S-PIV data. Main differences are due to different turbulence models and to the grid refinement adopted; overall, IIHR-GO is the more refined grid, ISIS-AMR shows finest resolution in the core of the vortices, whereas Xnavis-SA uses the coarsest grid.

Due to the rather coarse grid, Xnavis-SA computation shows the less intense and the more dissipated fore body keel vortex on all the sections reported. However, it also has to be noted that the

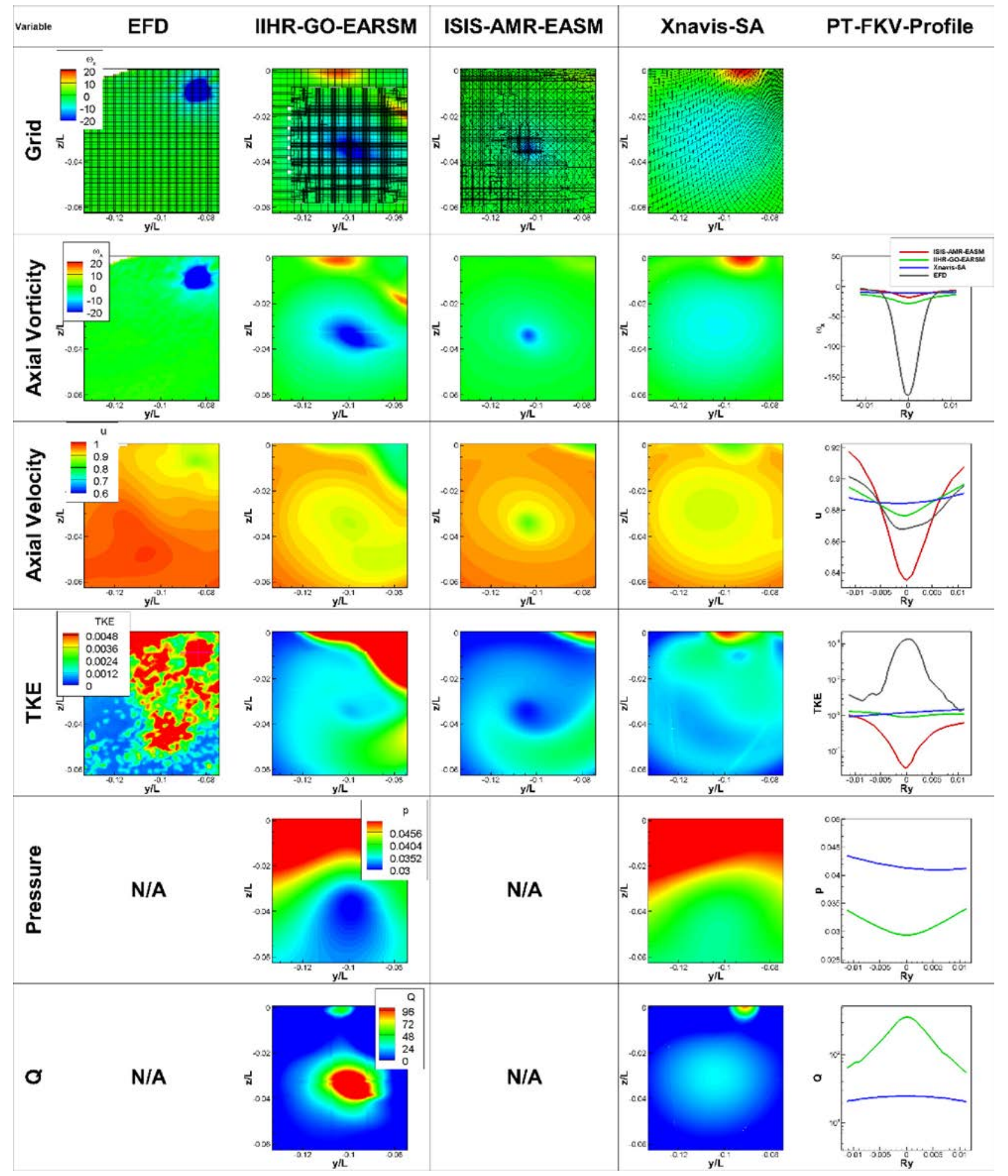

Fig. 15 Cross plane PT-FKV at P5. Fr $=0.4$ and $\beta=6$ deg. 
discrepancy for Xnavis-SA computation is also due to the turbulence model adopted, since the Spalart-Allmaras model has been demonstrated to be too dissipative. Computations which are using more refined grid and more sophisticated turbulence model (such as ISIS-EARSM-AMR and IIHR-EARSM-GO) provide sharper vorticity and velocity profiles, closer to EFD data; peak values are higher for ISIS computations than IIHR, i.e., in better agreement with EFD, but still very far (as already observed in the core vortex analysis). Xnavis-SA shows rather flat velocity and axial vorticity profile. For what TKE field concerns, IIHR-GO and Xnavis-SA provide similar flat profiles, whereas ISIS computation (using the EARSM turbulence model and grid refinement technique) shows a sharper profile with a large minimum value at the core of the vortex. On the contrary, EFD data report a maximum for the TKE in the core of the vortex. This discrepancy deserves an in-depth analysis which is left to future investigations. The prediction of very sharp profiles appears to be related to the use of the AMR technique.
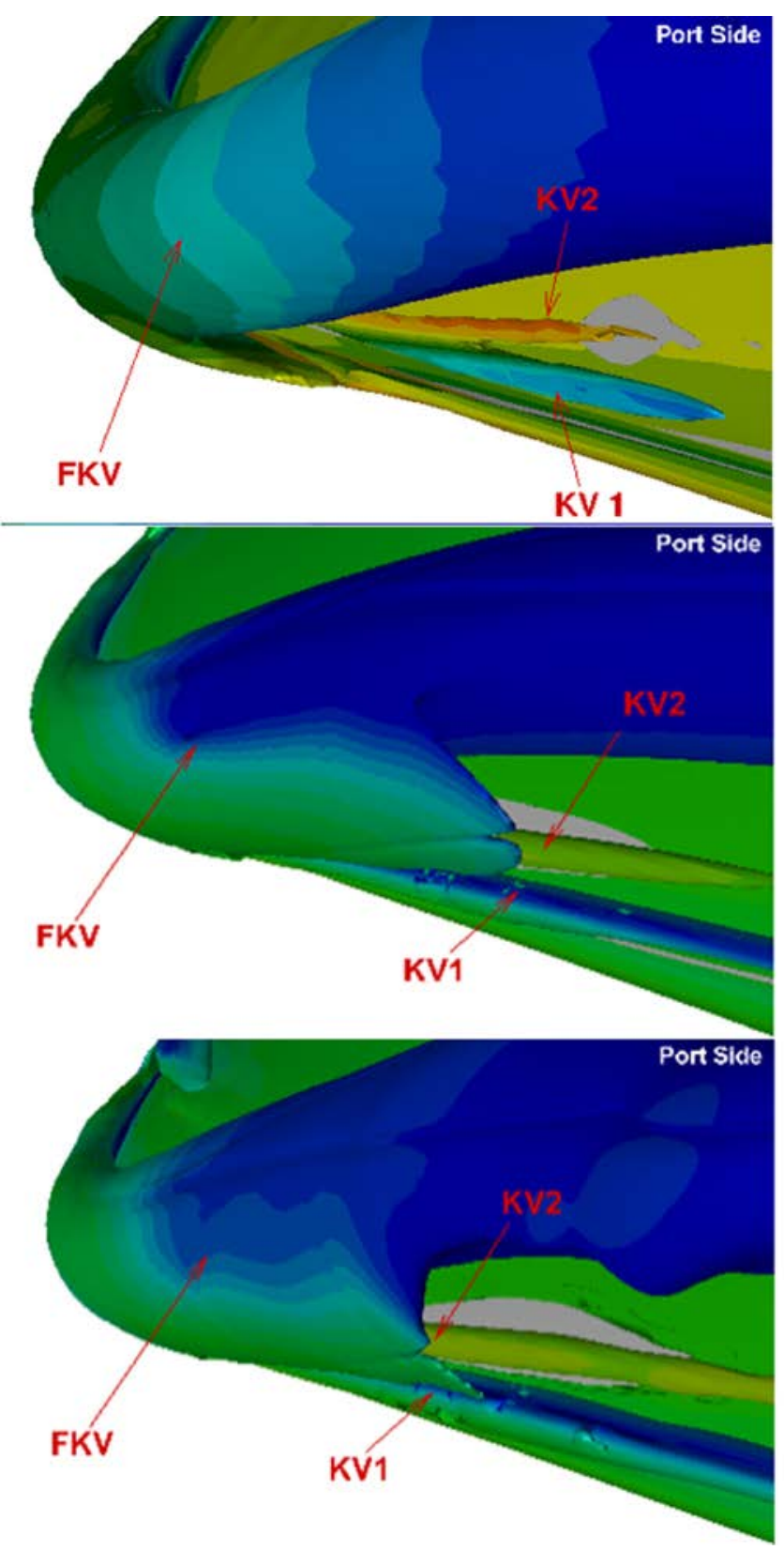

4.3.2 Keel Vortex. Along the keel of each hull, two other minor structures develop on the leeward side of the hull (see Figs. 6 and 8); these vortices are labeled as PT-KV1 and PT-KV2 on the port-side hull and ST-KV1 and ST-KV2 on the starboard hull. These vortical structures remain close to the hull surface, they are well reported by numerical simulations (see Figs. 16 and 17 for a detailed view), whereas were not captured by S-PIV measurements. One of the two vortices is co-rotating and the other is contra-rotating with respect to the FKV; the first one appears to be generated by the crossflow separation along the keel edge (i.e., similar to the FKV), the other one is generated by the downward flow induced by the FKV. The onset of these vortices is highlighted by converging streamlines forming a well-defined separation line. A common reattachment for both structures is evidenced by a dividing streamlines pattern. Limiting streamlines show that these vortices are generated from an open-type separation, which is clearly induced by the crossflow, i.e., without boundary layer separation. Computations provide rather weak structures which

Fig. 16 Detail of the $\mathrm{KVs}$ (only port side). $\mathrm{Fr}=0.4$ and $\beta=6 \mathrm{deg}$. For each submission: left, iso $Q=100$ colored with the normalized helicity; right, limiting streamlines. Top: Xnavis-SA; middle: IIHR-GO-SST; bottom: IIHR-GO-EARSM. 
are rapidly dissipated. An in-depth comparison between CFD submissions, reported in, reveals that differences are mainly due to grid resolution. As a matter of the fact, IIHR computations on GO predict stronger KVs, regardless of the turbulence model, whereas Xnavis-SA predicted weaker ones since the coarsest grid resolution. Grid resolution effects are confirmed comparing flow field predictions from ISIS-EARSM computations with and without AMR.

4.3.3 Free Surface Vortices and Wave Pattern. Due to the nonzero steady drift angle, breaking wave phenomenon followed by several ricochets develops on the windward side of hulls [56]; the breaking wave processes and the following ricochets generate two pairs of contra-rotating wave-induced vortices on the pressure side of each demi-hull (PT-FSV1 and ST-FSV1 and 2, and PT-FSV3/ST-FSV3 and 4, respectively, see Fig. 6). The generation of these vortices including the comparison between the computations is reported here; it has to be highlighted that S-PIV measurements do not provide any information about these structures, neither any wave pattern measurement is available. Breaking wave phenomenon occurs at all the tested conditions; in this section, the analysis is conducted for $\mathrm{Fr}=0.5-\beta=9 \mathrm{deg}$ condition for which the most energetic wave breaking and plunging phenomena occur and, as consequence, the strongest wave-induced vortices develop. Figure 18 shows the wave patterns estimation; only best submission from each institute (IIHR-DES-GW, Xnavis-SA, and ISIS-EARSM-AMR) has been reported.

In the figure, contour plot and iso-lines represent wave elevation with step sizes of $\Delta z=0.001 \mathrm{Lpp}$ and $\Delta z=0.002 \mathrm{Lpp}$, respectively; dashed lines represent negative elevation values. All the submissions are clearly capable to reproduce the main features of the wave pattern, with different levels of accuracy, depending on the grid resolution and the mathematical model adopted for the representation of the air/water interphase. In particular, a singlephase LS approach is adopted by Xnavis-SA and CFDShip-Iowa, the former in the framework of a finite volume discretization and the latter within a finite difference one. ISIS uses a two-phase volume of fluid $(\mathrm{VoF})$ approach. Xnavis-SA and ISIS computations provide rather strong wave breaking phenomena at the bow of both hulls as well as well-defined free surface scars on the windward sides, revealing the presence of wave-induced vortices. Wave breaking phenomena and the following ricochets are hardly seen in IIHR computations

Differences among numerical estimations do not seem to depend on grid resolution; in Fig. 18, computational grids adopted by the three selected computations are reported on plane P1 (close to the bow region, i.e., in the region where breaking waves and ricochets phenomena start). Clearly, IIHR adopts the most refined grid, whereas Xnavis-SA and ISIS use computational grids with similar refinement. Nevertheless, IIHR does not clearly show any wave breaking phenomenon. For ISIS computations, the advantage of using an AMR technique that allows breaking wave and ricochets to be captured with great accuracy with a much less number of total discretization points (similar results have been achieved by ECN/CNRS with AMR (7M) and without AMR (20M), see Ref. [11]) has to be highlighted.

As stated earlier, the onset of wave-induced vortices (FSVs) is consequent on the breaking wave and the following rebounds phenomena, i.e., different from the vortices previously analyzed, they are not generated by any flow separation. Progression of these vortices is driven by the mean flow, which is clearly (see Fig. 6) characterized by roll up of vortex pair and integration with fore
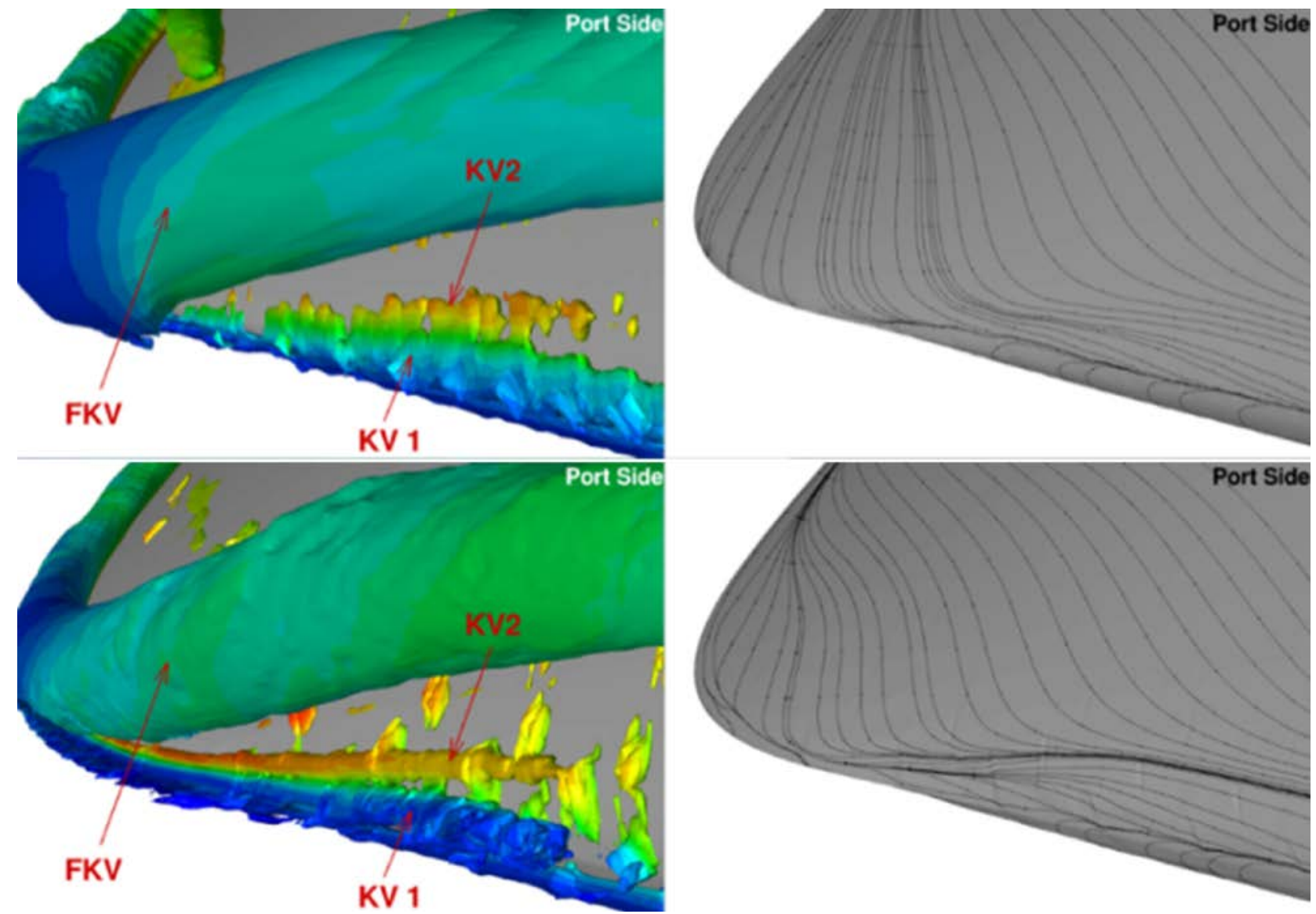

Fig. 17 Detail of the KVs (only port side). $\mathrm{Fr}=0.4$ and $\beta=6 \mathrm{deg}$. For each submission: left, iso $Q=100$ colored with the normalized helicity; right, limiting streamlines. Top: ISIS-EARSM; bottom: ISIS-EARSM-AMR. 
body keel vortices. In particular, the FSVs on the windward side of the port hull (i.e., PT-FSV3 and PT-FSV4) interact with STFKV around the stern.

In Fig. 19, cross sections of axial vorticity are shown on two transversal planes (P1 and P2), one close to the formation of the wave-induced vortices and the other around $0.75 \mathrm{Lpp}$. These sections well represent the evolution of the free surface vortices and, along grid resolutions reported in Fig. 18, highlight the main differences between the computational estimations. Xnavis-SA provides stronger and persistent vortices all along the hulls, which extend (see Fig. 6) up to the near wake. For the ISIS computations, similar generation is seen for FSVs, but with different progress with respect to Xnavis-SA. Pairs of negative and positive free surface vortices are still observable on plane P2 only for Xnavis-SA; for ISIS, wave-induced vortices remain close to the free surface, and, around amidships, they are advected toward the port-side hull, where they are definitely dissipated (see Fig. 18). It has to be noted that similar generation and progression for the wave-induced vortices is provided by ISIS computation adopting different turbulence models (nor reported here see Ref. [11], for more details). IIHR only provide an inception of wave breaking process at plane $\mathrm{P} 1$, without any formation of free surface vortices; consequently, for this computation, no wave-induced vortices are reported on plane $\mathrm{P} 2$.

\section{Large Drift Angle}

IIHR made some additional simulations, of particular interest are those conducted for the catamaran advancing straight ahead at low speed $(\mathrm{Fr}=0.30)$ with large drift angle $(\beta=24 \mathrm{deg})$. As reported in Table 13, these simulations have been performed with the $k \omega / k \varepsilon$-SST-DES and $k \omega / k \varepsilon$-SST-RANS models on G3 mesh (verification and validation of hydrodynamic loads for these simulations have already been reported in Sec. 3.3.2). In Fig. 20, an overview of the coherent structures is shown. Bottom views are presented in Fig. 21. Comparing these figures with the corresponding ones for smaller drift angle (Figs. 6 and 8), it is clear that similar coherent structures are generated. As expected, the fore-body keel vortices (PT-FKV and ST-FKV) and the keel vortices (ST-KV1-2, and PT-KV1-2) are stronger than those generated in the smaller drift cases. Also, the system of wave-induced vortices is similar with an additional free surface vortex on the leeward side of both hulls (PT-FSV5 and ST-FSV5).

From the bottom views of the vortical structures, comparison between DES and RANS simulations can be made. Clearly, DES simulation predicts stronger vortical structures than RANS computation; FKVs from DES are more refined and stronger, as well as the keel vortices $(\mathrm{KVs})$. From RANS computation, KVs are already dissipated around the stern, whereas, in the prediction with DES, they are still evident in the wake of the windward hull. Similarly, wave-induced vortices are more intense in the DES simulation. These observations confirm what has been already pointed out, i.e., DES, or more in general more sophisticated turbulence models, performs better in the prediction of the progression phase of the vortical structures (long life, less dissipated).

In Fig. 22, the numerical estimations of the wave patterns are compared with EFD measurements from BSHC [16]. Negligible differences between DES and RANS simulations are seen; in

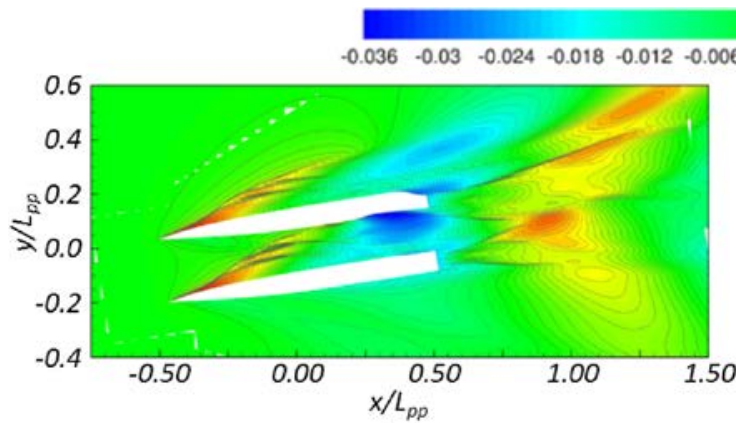

(a)

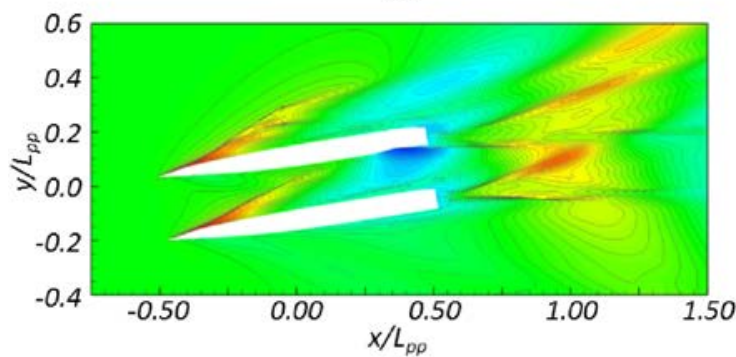

(c)

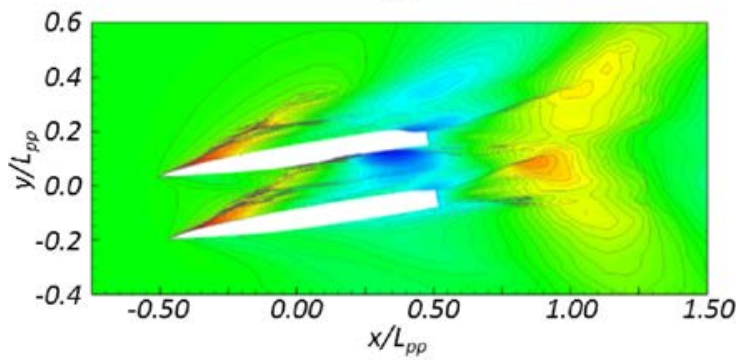

(e)

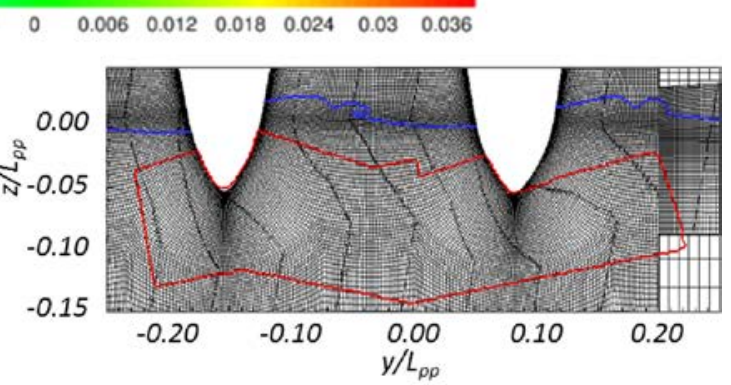

(b)

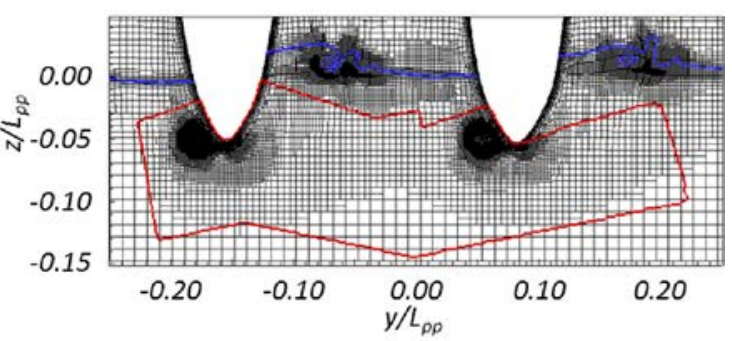

$(d)$

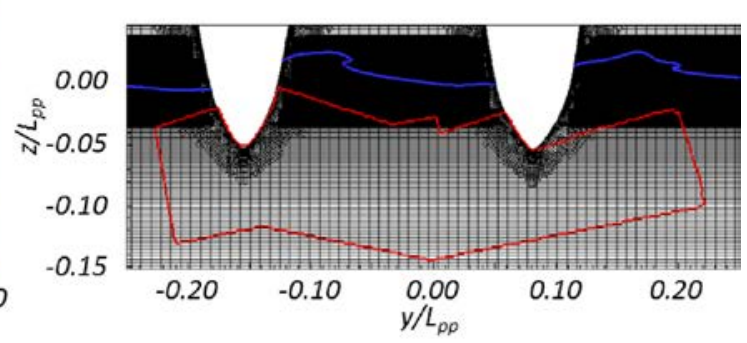

$(f)$

Fig. 18 Wave pattern (left column), $\mathrm{Fr}=0.5$ and $\beta=9$ and grid resolution on plane $\mathrm{P} 1$ (right column). (a) and (b) Xnavis; $(c)$ and (d) CFDShip-lowa; and $(e)$ and $(f)$ ISIS. 


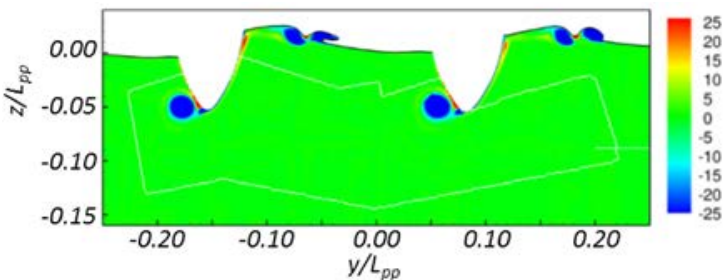

(a)

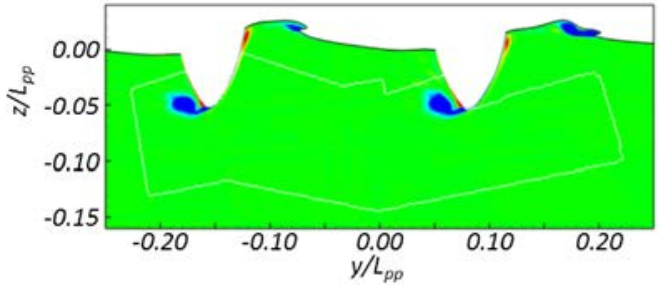

(c)

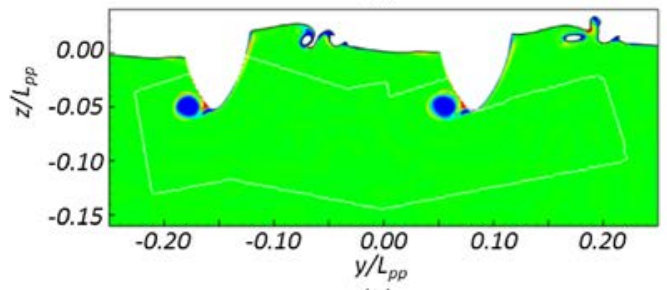

(e)

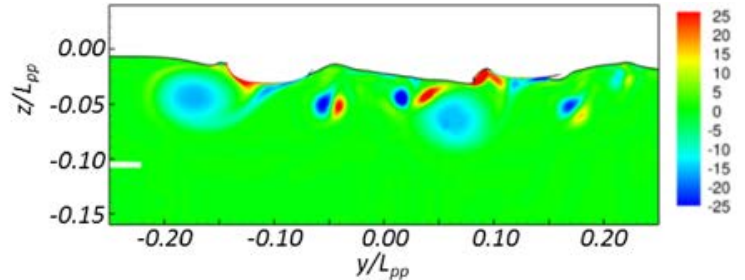

(b)

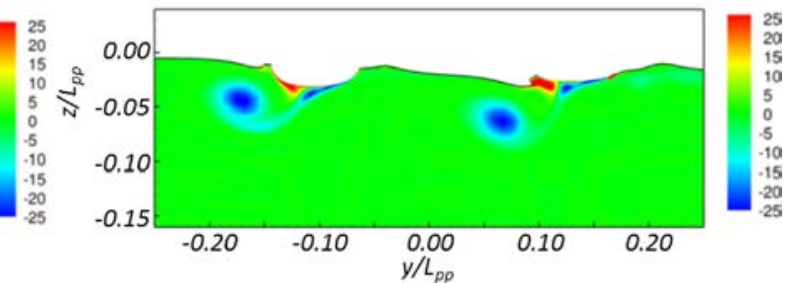

(d)

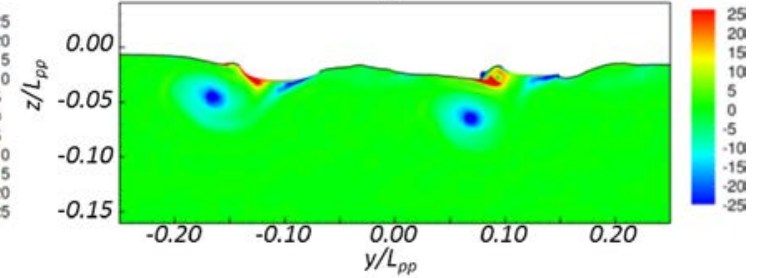

$(f)$

Fig. 19 Streamwise voticity, $\mathrm{Fr}=0.5$ and $\beta=9$ deg. Left column: plane P1; right column: plane P2. (a) and (b) Xnavis; (c) and ( $d$ ) CFDShip-lowa; and $(e)$ and ( $f$ ISIS.

particular in the region close to the catamaran, the solutions provide wave patterns in close agreement, some differences can be inferred in the wake region. These differences are clearly correlated with the differences seen in the predictions of the coherent structures. Some differences can be also observed when numerical estimations are compared with measurements; larger waves are predicted by the computations. The origin of the disagreement between CFD and EFD is not clear and deserves additional investigation.

\section{Conclusions and Final Recommendations}

In this paper, the assessment of CFD predictions has been performed for a Delft catamaran advancing in steady nonzero drift conditions; two speeds and two drift angles have been considered. Numerical computations have been performed by three institutions (CNR-INM, IIHR, and ECN/CNRS) from three countries (Italy, U.S., and France) using three different (in-house) codes, namely, Xnavis, CFDShip-Iowa, and ISIS. Computations have been performed on grids with different levels of resolutions, including the use of AMR technique. Several turbulence models have been used. For what air/water interface treatment concerns, a single-phase level set approach has been adopted by CFDShipIowa and Xnavis, whereas a volume of fluid method is implemented in ISIS. CFD study focused on validation of hydrodynamic loads prediction; validation of planar and volume flow field variables, including an in-depth analysis of the field variables along vortex core as it progresses downstream. The analysis involved also the study of the onset and the progression of the coherent structures detached from the hulls or generated by wave breaking phenomenon and the following splash up.

Hydrodynamic loads' estimations are in general good agreement among CFD submissions; differences between the estimations are within the numerical uncertainty. Differences are, obviously, ascribable to the turbulence model and/or grid refinement. In particular, those simulations using DES approach or anisotropic turbulence models have been proved to be superior to one equation isotropic model. Verification and validation have been assessed. In general, DES computations do not show good convergence properties. Better convergence properties, with low uncertainty, have been reported for RANS simulations. A rigorous validation of the numerical results could not be done, since hydrodynamic loads measurements were collected for a longer model and for speeds/drift angles different from those considered by the computations.

In the nonzero steady drift advancement, several vortices are generated along the two demi-hulls of the catamaran. All the numerical simulations, regardless of the grid refinement and the turbulence model adopted, are capable to capture the main features of the flow field. Some differences between numerical estimations have been seen both in the onset and in the progression phases of the main fore body keel vortices; also when comparing with EFD, some differences are seen, with the discrepancies increasing moving downstream. Differences among CFD in the onset region are mainly due to grid resolution, whereas moving downstream (i.e., for the progression of the main vortices) the

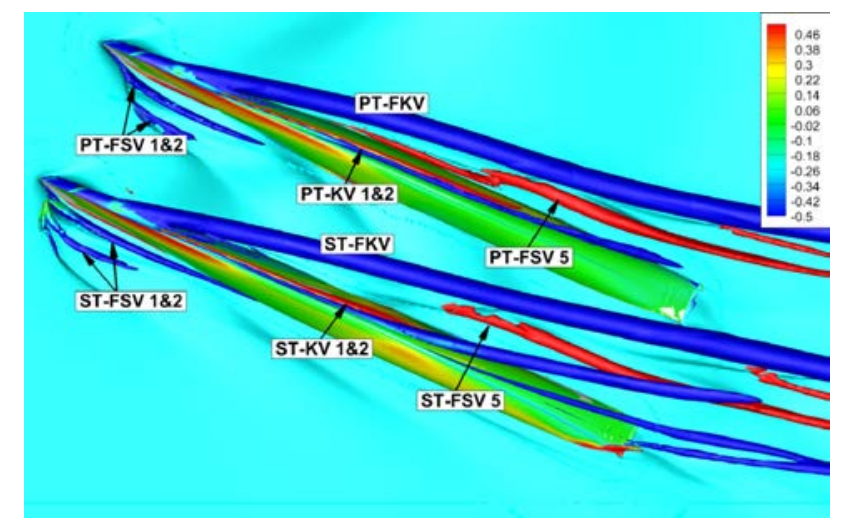

Fig. 20 Overview of the vortical structures (iso-surface of $Q=100$ colored with normalized helicity). $\beta=24 \mathrm{deg}$ and $\mathrm{Fr}=0.30$. IIHR-DES-G3. 


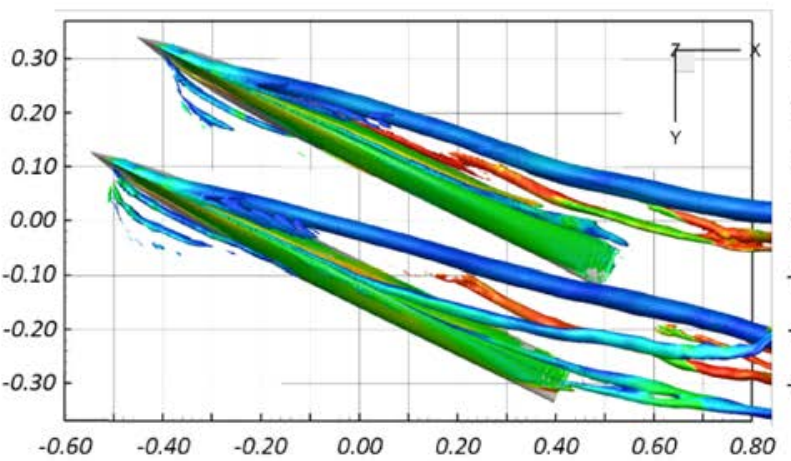

(a)

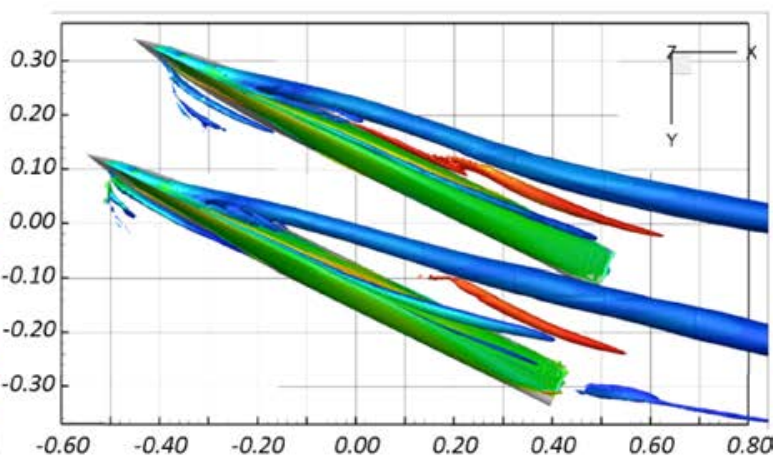

(b)

Fig. 21 IIHR-DES-G3. Iso-surfaces of $Q=100$ colored with normalized helicity ( $\mathrm{Fr}=0.3$ and $\beta=24$ deg). (a) IIHR-DES and (b) RANS on G3.

main reason can be ascribable to the adopted turbulence model. In general, anisotropic turbulence model and DES simulations provide better; however, simulations on more refined grid, in particular in the onset region, would provide more insight into turbulence model evaluation. Similar conclusions can be drawn for the vortex core analysis. In addition, a rather large under prediction is seen for the turbulent kinetic energy and the axial vorticity; the large disagreement is observed regardless of the turbulence model and the grid refinement.

Some differences have been also observed in the formation and the progression of wave-induced vortices; differences among numerical estimations are not ascribable to the grid resolution, rather on the model adopted to handle air/water interface (in the phase of the generation of the vortices) and on the interaction of the free surface model with the underlying turbulence model (in the progression phase).

To summarize, the current analysis has been highlighted that for the up-to-date CFD tools, weakness can be still ascribed to the lack in the design of the computational grid and in the deficiencies of the turbulence model closure. Therefore, efforts should be focused on (1) grid design to reduce the numerical dissipation, especially in the onset region of the vortices and (2) on the development of accurate turbulence closures to simulate the vortex flow dynamics.

From the in-depth analysis pursued in this research work, it is clear that future works should be focused on both experimental and numerical activities. For what experiments concerns, it is strongly recommended to collect measurements of global loads, providing reliable uncertainty assessment, for the same operative conditions at which flow measurement is acquired. Regarding velocity measurements, it would be desirable to have data with a high level of resolution and strong convergence properties. Further, it is deemed of paramount importance the collection of flow maps as much as close to the onset region, possibly with the inclusion of surface data (such as surface pressure, skin friction, and limiting streamlines). For what numerical activities concerns, it is strongly recommended to provide grid independent results; this would allow an accurate and an in-depth valuation of different

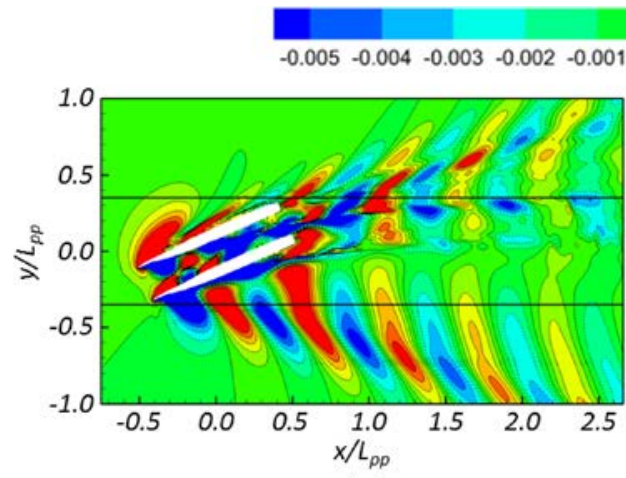

(a)

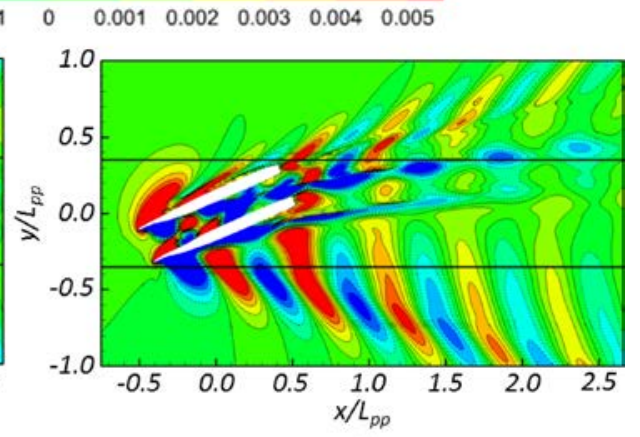

(b)

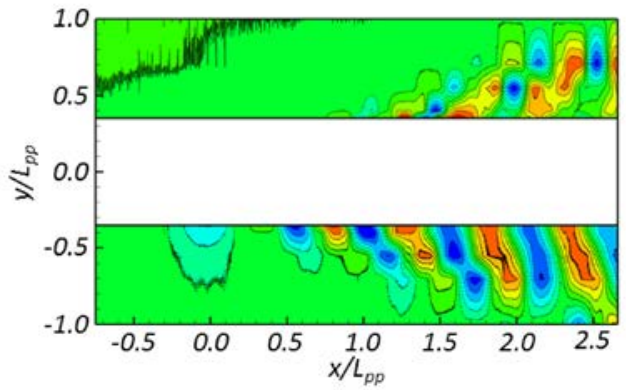

(c)

Fig. 22 Wave pattern ( $\mathrm{Fr}=\mathbf{0 . 3}$ and $\beta=24 \mathrm{deg})$. IIHR-DES (a) and RANS (b) on G3. Experimental results from BSHC [16] are reported in (c). 
turbulence models. Moreover, even if anisotropic turbulence models have been, in general, proven to be superior, numerical dissipation is clearly still too high for all the simulations submitted; highly resolved grid in the vortex core as well as more sophisticated closure models is deemed mandatory to accurately describe onset and progression of strong coherent structures. DES model, and in general, hybrid URANS/LES models shown to be highly promising; more investigation and development of hybrid models is recommended. Finally, in order to explain source of discrepancy and to guide the development of more sophisticated turbulence model, it is deemed of paramount importance to promote research activities focused on detailed analysis (either by experiments and high fidelity simulations) of TKE and vorticity budgets.

\section{Acknowledgment}

CNR-INM: The research has been partially supported by the Italian Flagship Project RITMARE, funded by the Italian Ministry of Education, Research Program 2011-2013 and by the U.S. Office of Naval research, in the framework of the NICOP projects (managed by Drs. Patrick Purtell, Ki-Han Kim, Thomas Fu, and Woei-Min Lin) "Complementary EFD and CFD Analysis of Calm Water Hydrodynamics and Large Amplitude Motion for HighSpeed Catamarans," Grant N00014-08-1-1037 and "Experimental Study of Static Drift and Stochastic Added Resistance for the Delft Catamaran," Grant N62909-12-1-7082.

ECN/CNRS: This work was granted access to the HPC resources of IDRIS under the allocations 2014-2A1309 and 2015-2A1308 made by GENCI (Grand Équipement National de Calcul Intensif).

IIHR, The University of Iowa: This research was sponsored by the Office of Naval Research under Grant N00014-01-1-0073 under the administration of Dr. Ki-Han Kim and Dr. Thomas Fu, whose support is greatly appreciated. Partial support to Timur Dogan was provided by the Naval Engineering Education Center (NEEC) of the U.S. Navy administered by Professor Steven Ceccio at the University of Michigan.

The authors wish to thank Dr. Ki-Han Kim for his thorough review of the paper.

\section{Funding Data}

- Grand Équipement National De Calcul Intensif (Grant No. 2014-2a1308; Funder ID: 10.13039/501100010190).

- Office of Naval Research (Grant No. N62909-12-1-7082; Funder ID: 10.13039/100000006).

- Office of Naval Research Global (Grant No. N00014-08-11037; Funder ID: 10.13039/100007297).

\section{References}

[1] Stern, F., Agdrup, K., Kim, S. Y., Cura-Hochbaum, A., Rhee, K. P., Quadvlieg, F., Perdon, P., Hino, T., Broglia, R., and Gorski, J., 2011, "Experience From SIMMAN 2008-The First Workshop on Verification and Validation of Ship Maneuvering Simulation Methods," J. Ship Res., 55(2), pp. 135-147.

[2] Simonsen, C. D., Stern, F., and Quadvlieg, F., 2014, "SIMMAN 2014: Workshop on V\&V of Ship Manoeuvering Simulation Methods," Workshop Proceedings, Copenhagen, Denmark, Dec. 8-10.

[3] Larsson, L., Stern, F., and Visonneau, M., 2013, Numerical Ship Hydrodynamics: An Assessment of the Gothenburg 2010 Workshop, Springer, Dordrecht, The Netherlands, p. 318.

[4] Larsson, L., Stern, F., Visonneau, M., Hino, T., Hirata, N., and Kim, J., 2018, "Tokyo 2015: A Workshop on CFD in Ship Hydrodynamics," Workshop Proceedings, Tokyo, Dec. 2-4.

[5] Marzi, J., and Broglia, R., 2019, "Hydrodynamic Tools in Ship Design," A Holistic Approach to Ship Design, A. Papanikolaou ed., Springer, Cham, Switzerland.

[6] Visonneau, M., Deng, G. B., Guilmineau, E., Queutey, P., and Wackers, J., 2016, "Local and Global Assessment of the Flow Around the Japan Bulk Carrier With and Without Energy Saving Devices at Model and Full Scale," 31st Symposium on Naval Hydrodynamics, Monterey, CA, Sept. 11-16.

[7] Stern, F., and Toxopeus, S. L., 2017, "Sea Facet Experimental and Computational Analysis Activities on Onset and Progression of Separation-Conclusions and Future Research," North Atlantic Treaty Organization Science \& Technology Organization, Brussels, Belgium, Technical Report No. TR-AVT183.
[8] Yoon, H., Gui, L., Bhushan, S., and Stern, F., 2014, "Tomographic PIV Measurements for a Surface Combatant at Straight Ahead and Static Drift Conditions," 30th Symposium on Naval Hydrodynamics, Hobart, Tasmania, Australia, Nov. 2-7.

[9] Bhushan, S., Yoon, H. Stern, F., Guilmineau, E., Visonneau, M., Toxopeus, S., Simonsen, C. D., Aram, S., Kim, S.-E., and Grigoropoulos, G., 2018, "Assessment of CFD for Surface Combatant 5415 at Straight Ahead and Static Drift $\beta=20$ deg," ASME J. Fluids Eng., 141(5), p. 051101.

[10] Abdel-Maksoud, M., Müller, V., Xing, T., Toxopeus, S., Stern, F., Petterson, K., Kim, S.-E., Eloot, K., Gietz, U., Schiller, P., and Rung, T., 2015, "Experimental and Numerical Investigations on Flow Characteristics of KVLCC2 at 30 deg Drift Angle," Fifth World Maritime Technology Conference, Providence, RI, Nov. 3-7.

[11] Broglia, R., Zaghi, S., Campana, E. F., Visonneau, M., Queutey, P., Dogan, T., Stern, F., and Milanov, E., 2017, "Chapter 6 - CFD Validation for Flow Separation Onset and Progression Using DELFT Catamaran 372 in Static Drift Conditions," Reliable Prediction of Separated Flow Onset and Progression for Air and Sea Vehicles, Summary Report of the NATO STO AVT-183 Task Group, Brussels, Belgium, Report No. RDP, STO-TR-AVT-183.

[12] Zlatev, Z. Z., Milanov, E., Chotukova, V., Sakamoto, N., and Stern, F., 2009 "Combined Model-Scale EFD-CFD Investigation of the Maneuvering Characteristics of a High Speed Catamaran," Tenth International Conference on Fast Sea Transportation (FAST), Athens, Greece, Oct. 5-9, pp. 449-462.

[13] Zlatev, Z. Z., Grigoropoulos, G., Milanov, E., and Stern, F., 2011, "CFD Studies of the Delft 372 Catamaran Bare Hull in Deep and Shallow Water," Specialists Meeting on Assessment of Stability and Control Prediction Methods for NATO Air \& Sea Vehicles, Portsdown, UK, Report No. NATO AVT-189.

[14] Milanov, E., Zlatev, Z. Z., Chotukova, V., and Stern, F., 2010, "Numerical and Experimental Prediction of the Inherent Course Stability of High Speed Catamaran in Deep and Shallow Water," 28th Symposium on Naval Hydrodynamics, Pasadena, CA, Sept. 12-17.

[15] Milanov, E., and Stern, F., 2012, "Static Drift Angle Test of Delft 372 Catamaran Bare Hull Model in Deep and Shallow Water," AVT-183 Meeting, Biarritz, France, Oct. 15-19.

[16] Milanov, E., and Georgiev, S., 2013, "Delft 373 Catamaran Maneuvering in Extreme Sea Conditions," Bulgarian Ship Hydrodynamics Centre, Varna, Bulgaria, Report No. KP102006/01

[17] Miozzi, M., 2011, "Towing and Self-Propulsion Tests of Waterjet Propeller DELFT-372 Catamaran," CNR-INSEAN, Rome, Italy, Report No. 2011-TR-011.

[18] Castiglione, T., Stern, F., Bova, S., and Kandasamy, M., 2011, "Numerical Investigation of the Seakeeping Behavior of a Catamaran Advancing in Regular Head Waves," Ocean Eng., 38(16), pp. 1806-1822.

[19] Bouscasse, B., Broglia, R., and Stern, F., 2013, "Experimental Investigation of a Fast Catamaran in Head Waves," Ocean Eng., 72, pp. 318-330.

[20] Broglia, R., Zaghi, S., and Di Mascio, A., 2011, "Numerical Simulation of Interference Effects for a High-Speed Catamaran,” J. Mar. Sci. Technol., 16(3), pp. 254-269.

[21] Broglia, R., Jacob, B., Zaghi, S., Stern, F., and Olivieri, A., 2013, "Experimental Investigation of Interference Effects for High-Speed Catamarans," Ocean Eng., 76, pp. 75-85.

[22] Zaghi, S., Broglia, R., and Di Mascio, A., 2011, "Analysis of the Interference Effects for High-Speed Catamarans by Model Tests and Numerical Simulations," Ocean Eng., 38(17-18), pp. 2110-2122.

[23] He, W., Castiglione, T., Kandasamy, M., and Stern, F., 2015, "Numerical Analysis of the Interference Effects on Resistance, Sinkage and Trim of a Fast Catamaran," J. Mar. Sci. Technol., 20(2), pp. 292-308.

[24] Milanov, E., Chotukova, V., Georgiev, F., and Stern, F., 2011, "EFD Studies on Delft 372 Catamaran Directional Stability and Maneuverability in Deep and Shallow Water," Specialists Meeting on Assessment of Stability and Control Prediction Methods for NATO Air \& Sea Vehicles, Portsdown, UK, Oct $12-14$, No. NATO AVT-189.

[25] Falchi, M., Felli, M., Grizzi, S., Aloisio, G., Broglia, R., and Stern, F., 2014, "SPIV Measurements Around the DELFT 372 Catamaran in Steady Drift," Exp. Fluids, 55(11), p. 1844.

[26] van't Veer, R., 1998, "Experimental Results of Motions and Structural Loads on the 372 Catamaran Model in Head and Oblique Waves," TU Delft, Delft, The Netherlands, Report No. 1130.

[27] van't Veer, R., 1998, "Experimental Results of Motions, Hydrodynamic Coefficients and Wave Loads on the 372 Catamaran Model," TU Delft, Delft, Netherlands, Report No. 1129.

[28] Di Mascio, A., Broglia, R., and Favini, B., 2001, A Second-order Godunov Type Scheme for Naval Hydrodynamics, Kluwer Academic/Plenum Publishers, Boston, MA, pp. 253-261.

[29] Di Mascio, A., Broglia, R., and Muscari, R., 2007, "On the Application of the Single-Phase Level Set Method to Naval Hydrodynamic Flows," Comput. Fluids, 36, pp. 868-886.

[30] Di Mascio, A., Broglia, R., and Muscari, R., 2009, "Prediction of Hydrodynamic Coefficients of Ship Hulls by High-Order Godunov-Type Methods," J. Mar. Sci. Technol., 14(1), pp. 19-29.

[31] Di Mascio, A., Muscari, R., and Broglia, R., 2006, "An Overlapping Grids Approach for Moving Bodies Problems," 16th International Offshore and Polar Engineering Conference (ISOPE), San Francisco, CA, May 28-June 2, Paper No. ISOPE-I-06-007.

[32] Broglia, R., Zaghi, S., Muscari, R., and Salvadore, F., 2014, "Enabling Hydrodynamics Solver for Efficient Parallel Simulations," International Conference on High Performance Computing \& Simulation (HPCS), Bologna, Italy, July 21-25, pp. 803-810. 
[33] Broglia, R., and Durante, D., 2018, "Accurate Prediction of Complex Free Surface Flow Around a High Speed Craft Using a Single-Phase Level Set Method," Comput. Mech., 62(3), pp. 421-437.

[34] Favini, B., Broglia, R., and Di Mascio, A., 1996, "Multigrid Acceleration of Second-Order ENO Schemes From Low Subsonic to High Supersonic Flows," Int. J. Numer. Methods Fluids, 23(6), pp. 589-606.

[35] Spalart, P. R., and Allmaras, S. R., 1994, A “One-Equation Turbulence Model for Aerodynamic Flows," La Rech. Aèrosp., 1, pp. 5-21.

[36] Huang, J., Carrica, P., and Stern, F., 2008, "Semi-Coupled Air/Water Immersed Boundary Approach for Curvilinear Dynamic Overset Grids With Application to Ship Hydrodynamics," Int. J. Numer. Methods Fluids, 58(6), pp. 591-624.

[37] Noack, R., 2005, "SUGGAR: A General Capability for Moving Body Overset Grid Assembly," AIAA Paper No. 2005-5117.

[38] Dogan, T. K., 2013, "URANS and DES for Delft Catamaran for Static Drift Conditions in Deep Water," M.S. thesis, University of Iowa, Iowa City, IA.

[39] Dogan, T. K., Sadat-Hosseini, H., and Stern, F., 2013, "DES of Delft Catamaran at Static Drift Condition,” Nav. Eng. J., 16(4), pp. 97-101.

[40] Menter, F., 1993, "Zonal Two Equation $\kappa-\omega$ Turbulence Models for Aerodynamic Flows," AIAA Paper No. 93-2906.

[41] Deng, G., and Visonneau, M., 1999, "Comparison of Explicit Algebraic Stress Models and Second-Order Turbulence Closures for Steady Flows Around Ships," Seventh International Conference on Numerical Ship Hydrodynamics, Nantes, France, July 19-22, pp. 1-15.

[42] Duvigneau, R., and Visonneau, M., 2003, "On the Role Played by Turbulence Closures in Hull Shape Optimization at Model and Full Scale," J. Mar. Sci. Technol., 8(1), pp. 11-25.

[43] Guilmineau, E., Chikhaoui, O., Deng, G. B., and Visonneau, M., 2013, "Cros Wind Effects on a Simplified Car Model by a DES Approach," Comput. Fluids, 78, pp. 29-40

[44] Queutey, P., and Visonneau, M., 2007, "An Interface Capturing Method for Free-Surface Hydrodynamic Flows," Comput. Fluids, 36(9), pp. 1481-1510.

[45] Leroyer, A., and Visonneau, M., 2005, "Numerical Methods for RANSE Simulations of a Self-Propelled Fish-Like Body,” J. Fluid Struct., 20(3), pp. 975-991.
[46] Deng, G. B., Queutey, P., Visonneau, M., and Salvatore, F., 2013, "Ship Propulsion Prediction With a Coupled RANSE-BEM Approach," Fifth Internationa Conference on Computational Methods in Marine Engineering (MARINE), Hamburg, Germany, May 29-31.

[47] Visonneau, M., Queutey, P., Wackers, J., Deng, G. B., and Guilmineau, E. 2012, "Sliding Grids and Adaptive Grid Refinement Applied to Ship Hydrodynamics," 29th Symposium on Naval Hydrodynamics, Gothenburg, Sweden, Aug. 26-31.

[48] Wackers, J., Deng, G. B., Guilmineau, E., Leroyer, A., Queutey, P., and Visonneau, M., 2014, "Combined Refinement Criteria for Anisotropic Grid Refinement in Free-Surface Flow Simulation," Comput. Fluids, 92, pp. 209-222.

[49] Broglia, R., Aloisio, G., Falchi, M., Grizzi, S., Zaghi, S., Felli, M., Miozzi, M. Pereira, F., Di Felice, F., and Stern, F., 2012, "Measurements of the Velocity Field Around the DELFT 372 Catamaran in Steady Drift," 29th Symposium on Naval Hydrodynamics, Gothenburg, Sweden, Aug. 29-31.

[50] Xing, T., and Stern, F., 2010, "Factors of Safety for Richardson Extrapolation," ASME J. Fluids Eng., 132(6), p. 061403.

[51] Xing, T., and Stern, F., 2011, "Closure to 'Discussion of 'Factors of Safety for Richardson Extrapolation',” ASME J. Fluids Eng., 133(11), p. 115502.

[52] Yoon, H. Y., 2009, "Force/Moment and Phase-Averaged Stereo PIV Flow Measurements for Surface Combatant in PMM Maneuvers," Ph.D. dissertation, The University of Iowa, Iowa City, IA.

[53] ASME, 2005,"Test Uncertainty," American Society of Mechanical Engineers, New York, Standard No. PTC 19.1-2005.

[54] Surana, A., Grunberg, O., and Haller, G., 2006, "Exact Theory of ThreeDimensional Flow Separation-Part I: Steady Separation,” J. Fluid Mech., 564, pp. 57-103.

[55] Délery, J. M., 2001, "Robert Legendre and Henri Werlé: Toward the Elucidation of Three-Dimensional Separation,” Annu. Rev. Fluid Mech., 33(1), pp. 129-154.

[56] Olivieri, A., Pistani, F., Wilson, R., Campana, E. F., and Stern, F., 2007, "Scars and Vortices Induced by Ship Bow and Shoulder Wave Breaking," ASME. J. Fluids Eng., 129(11), pp. 1445-1459. 